\title{
RESOLUTIONS OF NEWTON NON-DEGENERATE MIXED POLYNOMIALS OF STRONGLY POLAR NON-NEGATIVE MIXED WEIGHTED HOMOGENEOUS FACE TYPE
}

\author{
SACHIKO SAITO AND KOSEI TAKASHIMIZU
}

\begin{abstract}
Let $f(\boldsymbol{z}, \overline{\boldsymbol{z}})$ be a convenient Newton non-degenerate mixed polynomial with strongly polar nonnegative mixed weighted homogeneous face functions. We consider a convenient regular simplicial cone subdivision $\Sigma^{*}$ which is admissible for $f$ and take the toric modification $\hat{\pi}: X \rightarrow \mathbb{C}^{n}$ associated with $\Sigma^{*}$. We show that the toric modification resolves topologically the singularity of the mixed hypersurface germ defined by $f(\boldsymbol{z}, \overline{\boldsymbol{z}})$ under the $\operatorname{Assumption}(*)$ (Theorem [32). This result is an extension of the first part of Theorem 11 (4]) by M. Oka, which studies strongly polar positive cases, to strongly polar non-negative cases. We also consider some typical examples (\$9).
\end{abstract}

\section{INTRODUCTION}

A mixed analytic function (or mixed function) $f(\boldsymbol{z}, \overline{\boldsymbol{z}})$ on a neighborhood $U$ of $\mathbf{0}$ in $\mathbb{C}^{n}$ is defined to be $f(\boldsymbol{z}, \overline{\boldsymbol{z}}):=F(\boldsymbol{z}, \overline{\boldsymbol{z}})$, where $F(\boldsymbol{z}, \boldsymbol{w})$ on $U \times U$ is a complex valued holomorphic function with complex $2 n$ variables. For a mixed function $f(\boldsymbol{z}, \overline{\boldsymbol{z}})$ on $U$, we consider the mixed hypersurface $V:=f^{-1}(0)$ as a germ at $\mathbf{0}$. We study resolutions of the singularity $(V, \mathbf{0})$ by toric modifications when $\mathbf{0}$ is an isolated mixed singular point (see \$2) of $V$. In [4, M. Oka obtained Theorem 11 concerning topological resolutions of the isolated singularity $\mathbf{0} \in V:=f^{-1}(0)$ by toric modifications under the assumption that $(f, \mathbf{0})$ is a convenient and Newton non-degenerate mixed polynomial germ of strongly polar positive mixed weighted homogeneous face type. Theorem 11 of [4] (see also Theorem 9.19 of [5]) is a generalization of Varchenko's famous Lemma 10.3 ([12]) on holomorphic function germs to mixed polynomial germs.

The main purpose of this paper is to prove an extension (Theorem 32) of the first part $((1)$ and (2)) of Theorem 11 of [4] to strongly polar non-negative mixed weighted homogeneous face type cases. Moreover, our Theorem 32 includes the information about the differentiability $\left(C^{s}\right.$-class, where $\left.s \geq 0\right)$ of the strict transform $\tilde{V}$ of $V$. At present we do not get any extensions of the latter part (3) of Theorem 11 ([4]), which is a result on the zeta functions of the Milnor fibrations of mixed polynomials. In strongly polar non-negative mixed weighted homogeneous face type cases, we do not always have the Milnor fibrations. The simplest example is the mixed function $f: \mathbb{C} \rightarrow \mathbb{C}, f(z, \bar{z}):=z \bar{z}$. This function does not have the Milnor fibration but the zero set $f^{-1}(0)$ still coincides with the holomorphic case: $g(z):=z^{2}$. In $\$ 9$ of this paper we consider and calculate some typical examples including the mixed polynomial $\left(z_{1}^{4}+z_{2}^{3}\right) \overline{\left(z_{1}^{2}+z_{2}^{3}\right)}$. Before the studies of mixed functions, a real function of type $f \bar{g}$ had been studied by A. Pichon and J. Seade [7]. They mentioned that it has the Milnor fibration under the assumption that the multiplicities of $f$ and $g$ do not coincide on each of the exceptional divisors. The coincidence of the multiplicities means that the polar degree of the corresponding face function of $f \bar{g}$ is 0 . See also [6] and [8].

In $\$ 2$ and $\$ 3$ we introduce some basic terms, namely, mixed functions, mixed polynomials, mixed critical points, the (radial) Newton polyhedron of a mixed function germ, the face functions of a mixed function germ, and so on. In $\$$ we give the definitions of mixed function germs of strongly polar positive (respectively, of strongly polar non-negative) mixed weighted homogeneous face type. In \$5 we describe the notion of Newton non-degeneracy of mixed function germs. We also mention about strong Newton non-degeneracy. Although we do not use strong Newton non-degeneracy in the statement of our main Theorem 32, we should note that for mixed weighted homogeneous polynomials (Definition 10) of non-zero polar degree,

2010 Mathematics Subject Classification. 14P05, 32S45, 32S55.

Key words and phrases. mixed polynomial, strongly mixed weighted homogeneous, Newton non-degenerate, toric modification. 
Newton non-degeneracy implies strong Newton non-degeneracy. See Remark [16, In $\$ 6$ and $\$ 7$ we moreover introduce important terms, regular simplicial cone subdivisions, toric modifications, and convenient mixed function germs. We prepare the key Propositions 21] 23, Lemma 29] and Proposition 30, which we use in the proof of the main Theorem 32 .

In $\$ 8$ we state the main Theorem 32 . The assertion (ii) of Theorem 32 includes the differentiability of the strict transform germ $\tilde{V}$. For the proof of (ii), we prepare Lemma 31, which describes the differentiability of functions in the form of $u^{r+s} / \bar{u}^{r}$.

In the statement of Theorem 32 (i), we put the assumption that "the vertices of $\tau$ are all strictly positive". We at present cannot prove (i) without this assumption. Following (i), we put Assumption $(*)$ in the statement of Theorem 32 (ii). The reason is as follows: We prove (ii) by using the argument in the proof of (i). For an arbitrary point $\hat{\boldsymbol{u}}^{0} \in \tilde{V} \cap\left(\bigcup_{P \gg 0} \hat{E}(P)\right)$, there exist some vertices $P_{1}, \ldots, P_{\kappa}(1 \leq \kappa \leq n)$ and an $n$-dimensional cone $\sigma=\left(P_{1}, \ldots, P_{n}\right)$ for which the toric chart $\mathbb{C}_{\sigma}^{n}$ contains $\hat{\boldsymbol{u}}^{0}=(\underbrace{0, \ldots, 0}_{\kappa}, u_{\kappa+1}^{0}, \ldots, u_{n}^{0})$ and $u_{\kappa+1}^{0} \neq 0, \ldots, u_{n}^{0} \neq 0$. If some of $P_{1}, \ldots, P_{\kappa}$ are not strictly positive, then we cannot apply (i) to the cone $\left(P_{1}, \ldots, P_{\kappa}\right)$. Therefore we assume Assumption $\left(^{*}\right)$ in (ii). It will be interesting to study whether $\operatorname{Assumption}(*)$ can be removed or not.

In the last section 9 we investigate the mixed polynomials $z_{1}^{4} \bar{z}_{1}^{2}+z_{1}^{a} \bar{z}_{1}^{4-a} z_{2}^{b} \bar{z}_{2}^{3-b}+\bar{z}_{1}^{2} z_{2}^{3}+z_{2}^{3} \bar{z}_{2}^{3}$ including $\left(z_{1}^{4}+z_{2}^{3}\right) \overline{\left(z_{1}^{2}+z_{2}^{3}\right)}$ in detail.

\section{Mixed AnALytiC FunCtions And MiXed Polynomials}

Let $U$ be a neighborhood of $\mathbf{0}$ in $\mathbb{C}^{n}$. We assume $\bar{U}=U$, where $\overline{\boldsymbol{z}}$ stands for the complex conjugate $\left(\overline{z_{1}}, \ldots, \overline{z_{n}}\right)$ of $\boldsymbol{z}=\left(z_{1}, \ldots, z_{n}\right) \in \mathbb{C}^{n}$. For a complex valued holomorphic function $F(\boldsymbol{z}, \boldsymbol{w})$ on $U \times U$ with complex $2 n$ variables, we set

$$
f(\boldsymbol{z}, \overline{\boldsymbol{z}}):=F(\boldsymbol{z}, \overline{\boldsymbol{z}})
$$

which is defined over $U$. We call it a mixed analytic function (or mixed function) $f$ on $U$. We assume that $F(\mathbf{0}, \mathbf{0})=0$ throughout this paper, and hence,

$$
f(\mathbf{0})=0 .
$$

Let

$$
F(\boldsymbol{z}, \boldsymbol{w})=\sum_{\nu, \mu} c_{\nu, \mu} \boldsymbol{z}^{\nu} \boldsymbol{w}^{\mu}
$$

be the Taylor expansion of $F$ at $(\mathbf{0}, \mathbf{0})$, where $\nu=\left(\nu_{1}, \ldots, \nu_{n}\right), \mu=\left(\mu_{1}, \ldots, \mu_{n}\right), \nu_{i} \geq 0, \mu_{j} \geq 0, \quad \boldsymbol{z}^{\nu}:=$ $z_{1}^{\nu_{1}} \cdots z_{n}^{\nu_{n}}, \quad \boldsymbol{w}^{\mu}:=w_{1}^{\mu_{1}} \cdots w_{n}^{\mu_{n}}$. Then we have $c_{\mathbf{0}, \mathbf{0}}=0$ and

$$
f(\boldsymbol{z}, \overline{\boldsymbol{z}})=\sum_{\nu, \mu} c_{\nu, \mu} \boldsymbol{z}^{\nu} \overline{\boldsymbol{z}}^{\mu}
$$

We call $f(\boldsymbol{z}, \overline{\boldsymbol{z}})$ a mixed polynomial when the number of monomials $c_{\nu, \mu} \boldsymbol{z}^{\nu} \overline{\boldsymbol{z}}^{\mu}, c_{\nu, \mu} \neq 0$ is finite.

For a mixed function $f$ on $U$, let us consider

$$
V:=f^{-1}(0) \quad(\subset U)
$$

which we call a mixed hypersurface. We set $z_{j}=x_{j}+i y_{j}$ and consider the real valued functions

$$
g(\boldsymbol{x}, \boldsymbol{y}):=\Re f(\boldsymbol{z}, \overline{\boldsymbol{z}}), \quad h(\boldsymbol{x}, \boldsymbol{y}):=\Im f(\boldsymbol{z}, \overline{\boldsymbol{z}})
$$

with real $2 n$ variables $(\boldsymbol{x}, \boldsymbol{y})$. Then we have $f(\boldsymbol{z}, \overline{\boldsymbol{z}})=g(\boldsymbol{x}, \boldsymbol{y})+i h(\boldsymbol{x}, \boldsymbol{y})$, and

$$
V=\{(\boldsymbol{x}, \boldsymbol{y}) \in U \mid g(\boldsymbol{x}, \boldsymbol{y})=h(\boldsymbol{x}, \boldsymbol{y})=0\} .
$$

Thus $V$ is a real analytic variety in $U\left(\subset \mathbb{R}^{2 n}\right)$. 
Definition 1. Let $f(\boldsymbol{z}, \overline{\boldsymbol{z}})$ be a mixed analytic function on $U\left(\subset \mathbb{C}^{n}\right)$. We say $\boldsymbol{a}=\left(a_{1}, \ldots, a_{n}\right) \in U$ is a mixed critical point (or a mixed singular point) of $f$ if the rank of the differential map $(d f)_{\boldsymbol{a}}: T_{\boldsymbol{a}} \mathbb{C}^{n} \rightarrow$ $T_{f(\boldsymbol{a})} \mathbb{C} \cong T_{f(\boldsymbol{a})} \mathbb{R}^{2}$ is less than 2. We also say $\boldsymbol{a}$ is a mixed singular point of the mixed hypersurface $f^{-1}(f(\boldsymbol{a}, \overline{\boldsymbol{a}}))$ when $\boldsymbol{a}$ is a mixed critical point of $f$. We say $\boldsymbol{a} \in U$ is a mixed regular point of $f$ if it is not a mixed critical point of $f$.

We set $\partial f:=\left(\frac{\partial f}{\partial z_{1}}, \ldots, \frac{\partial f}{\partial z_{n}}\right), \quad \bar{\partial} f:=\left(\frac{\partial f}{\partial \bar{z}_{1}}, \ldots, \frac{\partial f}{\partial \bar{z}_{n}}\right)$, where

$$
\frac{\partial}{\partial z_{j}}=\frac{1}{2}\left(\frac{\partial}{\partial x_{j}}-i \frac{\partial}{\partial y_{j}}\right), \quad \frac{\partial}{\partial \bar{z}_{j}}=\frac{1}{2}\left(\frac{\partial}{\partial x_{j}}+i \frac{\partial}{\partial y_{j}}\right)
$$

Equivalently, we have

$$
\frac{\partial}{\partial x_{j}}=\frac{\partial}{\partial z_{j}}+\frac{\partial}{\partial \bar{z}_{j}}, \quad \frac{\partial}{\partial y_{j}}=i\left(\frac{\partial}{\partial z_{j}}-\frac{\partial}{\partial \bar{z}_{j}}\right) .
$$

Proposition 2 (Oka 2], Proposition 1, [5], Proposition 8.1). The following two conditions are equivalent:

(1) $\boldsymbol{a}=\left(a_{1}, \ldots, a_{n}\right)$ is a mixed critical point of $f$.

(2) There exists a complex number $\alpha$ with $|\alpha|=1$ which satisfies $\overline{\partial f(\boldsymbol{a}, \overline{\boldsymbol{a}})}=\alpha \bar{\partial} f(\boldsymbol{a}, \overline{\boldsymbol{a}})$.

Corollary 3. If

$$
\left|\frac{\partial f}{\partial z_{j}}(\boldsymbol{a}, \overline{\boldsymbol{a}})\right| \neq\left|\frac{\partial f}{\partial \overline{z_{j}}}(\boldsymbol{a}, \overline{\boldsymbol{a}})\right|
$$

for some $j(1 \leq j \leq n)$, then $\boldsymbol{a}$ is a mixed regular point of $f$.

\section{3. (RAdial) NEWTON POlyhedrons ANd their FACES}

We first define $\mathbb{R}_{+}^{n}:=\left\{\left(x_{1}, \ldots, x_{n}\right) \mid x_{i} \geq 0\right\}$. Let $(f, \mathbf{0})$ be the germ of a mixed function

$$
f(\boldsymbol{z}, \overline{\boldsymbol{z}})=\sum_{\nu, \mu} c_{\nu, \mu} \boldsymbol{z}^{\nu} \overline{\boldsymbol{z}}^{\mu}
$$

at $\mathbf{0} \in \mathbb{C}^{n}$. Let

$$
\Gamma_{+}(f)
$$

be the convex hull of the set

$$
\bigcup_{c_{\nu, \mu} \neq 0}(\nu+\mu)+\mathbb{R}_{+}^{n}
$$

which we call the (radial) Newton polyhedron of the germ $(f, \mathbf{0})$ of a mixed function $f$ at $\mathbf{0}$.

Definition 4 (face of $\Gamma_{+}(f)$ ). For a "weight vector"

$$
P={ }^{t}\left(p_{1}, \ldots, p_{n}\right)(\neq \mathbf{0}) \in N_{\mathbb{R}}^{+}:=\mathbb{R}_{+}^{n},
$$

let

$$
d(P)
$$

be the minimum value of the linear function

$$
P: \Gamma_{+}(f) \rightarrow \mathbb{R}, P(\xi):=\sum_{j=1}^{n} p_{j} \xi_{j} \quad(\in \mathbb{R}),
$$

where $\xi=\left(\xi_{1}, \ldots, \xi_{n}\right) \in \Gamma_{+}(f)$. We set

$$
\Delta(P):=\left\{\xi \in \Gamma_{+}(f) \mid P(\xi)=d(P)\right\}
$$

which we call a face of $\Gamma_{+}(f)$. Note that $\Delta(P) \neq \emptyset$ by its definition. If $\operatorname{dim} \Delta(P)=n-1$, then $P$ is unique up to multiplications of positive real numbers.

Definition 5 (strictly positive weight vector). We say a weight vector $P={ }^{t}\left(p_{1}, \ldots, p_{n}\right)$ is strictly positive if $p_{i}>0$ for every $i(=1, \ldots, n)$. We write $P \gg 0$ if $P$ is strictly positive. 
Note that a face $\Delta$ of $\Gamma_{+}(f)$ is compact if and only if $\Delta=\Delta(P)$ for some strictly positive weight vector $P$. For the proof, for example, see [10].

Definition 6 (the (radial) Newton boundary $\Gamma(f)$ ). Let $\Gamma(f)$ be the union of all compact faces of $\Gamma_{+}(f)$, which we call the (radial) Newton boundary of the germ $(f, \mathbf{0})$ of a mixed function $f$.

Definition 7 (face function). For a compact face $\Delta(P)(\subset \Gamma(f))$, we define

$$
f_{\Delta(P)}(\boldsymbol{z})\left(\text { or } f_{P}(\boldsymbol{z})\right):=\sum_{\mu+\nu \in \Delta(P)} c_{\nu, \mu} \boldsymbol{z}^{\nu} \overline{\boldsymbol{z}}^{\mu}
$$

which we call a face function (or face polynomial) of a mixed function germ $(f, \mathbf{0})$ (cf. [5], p.78).

\section{RADiAL AND POLAR WEIGHTED HOMOGENEOUS POLYNOMIALS}

Definition 8 ([5], p.182, [4]). A mixed polynomial $f(\boldsymbol{z}, \overline{\boldsymbol{z}})=\sum_{\nu, \mu} c_{\nu, \mu} \boldsymbol{z}^{\nu} \overline{\boldsymbol{z}}^{\mu}$ is called radially weighted homogeneous if there exists a weight vector

$$
Q={ }^{t}\left(q_{1}, \ldots, q_{n}\right)(\neq \mathbf{0}) \in N_{\mathbb{R}}^{+}:=\mathbb{R}_{+}^{n}
$$

and a positive integer $d_{r}(>0)$ such that

$$
c_{\nu, \mu} \neq 0 \Longrightarrow \sum_{i=1}^{n} q_{i}\left(\nu_{i}+\mu_{i}\right)=d_{r} .
$$

We call $d_{r}$ the radial degree of $f$, and define

$$
\operatorname{rdeg}_{Q} f:=d_{r} .
$$

A mixed polynomial $f(\boldsymbol{z}, \overline{\boldsymbol{z}})=\sum_{\nu, \mu} c_{\nu, \mu} \boldsymbol{z}^{\nu} \overline{\boldsymbol{z}}^{\mu}$ is called polar weighted homogeneous if there exists a weight vector

$$
P={ }^{t}\left(p_{1}, \ldots, p_{n}\right)(\neq \mathbf{0}) \in N_{\mathbb{R}}^{+}:=\mathbb{R}_{+}^{n}
$$

and an integer $d_{p}(>0,0$ or $<0)$ such that

$$
c_{\nu, \mu} \neq 0 \Longrightarrow \sum_{i=1}^{n} p_{i}\left(\nu_{i}-\mu_{i}\right)=d_{p} .
$$

We call $d_{p}$ the polar degree of $f$, and define

$$
\operatorname{pdeg}_{P} f:=d_{p}
$$

Remark 9. Since we assume $f(\mathbf{0})=0$, every face function (Definition 7) $f_{P}(\boldsymbol{z})=f_{\Delta(P)}(\boldsymbol{z})$, where we take a strictly positive $P$, of a mixed function germ $(f, \mathbf{0})$ is a radially weighted homogeneous polynomial of radial degree $d(P)(>0)$ with respect to the weight vector $P$.

Definition 10 ([5], pp.182-184). We say a mixed polynomial $f(\boldsymbol{z}, \overline{\boldsymbol{z}})$ is a mixed weighted homogeneous polynomial 11 if it is both radially and polar 2 weighted homogeneous.

Definition 11 (cf. [5],p.183, 4],p.174). We say a mixed weighted homogeneous polynomial $f(\boldsymbol{z}, \overline{\boldsymbol{z}})$ is a strongly mixed weighted homogeneous polynomial if $f$ is radially and polar weighted homogeneous with respect to the same weight vector $P$. Furthermore, $f$ is called a strongly polar positive (respectively, strongly polar non-negative) mixed weighted homogeneous polynomial if $\operatorname{pdeg}_{P} f>0\left(\operatorname{respectively,~} \operatorname{pdeg}_{P} f \geq 0\right)$.

Definition 12 (cf. [5],Definition 9.18, 4],p.174). Let $(f, \mathbf{0})$ be a mixed function germ at $\mathbf{0} \in \mathbb{C}^{n}$.

(1) The germ $(f, \mathbf{0})$ of a mixed function $f(\boldsymbol{z}, \overline{\boldsymbol{z}})$ at $\mathbf{0} \in \mathbb{C}^{n}$ is called of strongly mixed weighted homogeneous face type if the face function $f_{\Delta}(\boldsymbol{z}, \overline{\boldsymbol{z}})$ is a strongly mixed weighted homogeneous polynomial (Definition 11) for every compact face $\Delta$.

\footnotetext{
${ }^{1}$ We use the definition in [5].

${ }^{2}$ The corresponding weight vectors $Q$ and $P$ are possibly different.
} 
(2) The germ $(f, \mathbf{0})$ of a mixed function $f(\boldsymbol{z}, \overline{\boldsymbol{z}})$ at $\mathbf{0} \in \mathbb{C}^{n}$ is called of strongly polar positive (respectively, of strongly polar non-negative) mixed weighted homogeneous face type if for every 3 compact face $\Delta$, the face function $f_{\Delta}(\boldsymbol{z}, \overline{\boldsymbol{z}})$ is a strongly polar positive (respectively, strongly polar non-negative) mixed weighted homogeneous polynomial (Definition 11) with respect to some strictly positive weight vector $P$ with $\Delta=\Delta(P)$.

Remark 13. For mixed function germs of strongly polar non-negative mixed weighted homogeneous face type, Proposition 30 is important.

\section{NEWTON NON-DEGENERACY}

Definition 14 ([3], p.6, Definition 3, [5], p.80 and pp.181-182). Let $(f, 0)$ be the germ of a mixed function $f$ at $\mathbf{0} \in \mathbb{C}^{n}$.

(1) We say $(f, \mathbf{0})$ is Newton non-degenerate over a compact face $\Delta(\subset \Gamma(f))$ if 0 is not a mixed critical value of the face function $f_{\Delta}: \mathbb{C}^{* n} \rightarrow \mathbb{C}$. (In particular, if $f_{\Delta}^{-1}(0) \cap \mathbb{C}^{* n}=\emptyset$, then 0 is not a mixed critical value of the face function $f_{\Delta}: \mathbb{C}^{* n} \rightarrow \mathbb{C}$.)

(2) Let $\Delta(\subset \Gamma(f))$ be a compact face with $\operatorname{dim} \Delta \geq 1$. We say $(f, \mathbf{0})$ is strongly Newton non-degenerate over $\Delta$ if the face function $f_{\Delta}: \mathbb{C}^{* n} \rightarrow \mathbb{C}$ has no mixed critical points and $f_{\Delta}: \mathbb{C}^{* n} \rightarrow \mathbb{C}$ is surjective onto $\mathbb{C}$.

(3) Let $\Delta(\subset \Gamma(f))$ be a compact face with $\operatorname{dim} \Delta=0$, that is, $\Delta$ is a vertex of $\Gamma_{+}(f)$. We say $(f, \mathbf{0})$ is strongly Newton non-degenerate over $\Delta$ if the face function $f_{\Delta}: \mathbb{C}^{* n} \rightarrow \mathbb{C}$ has no mixed critical points 4 .

Definition 15 ([5], p.80 and p.182). We say the germ $(f, \mathbf{0})$ of a mixed function $f$ at $\mathbf{0} \in \mathbb{C}^{n}$ is Newton nondegenerate (respectively, strongly Newton non-degenerate) if $(f, \mathbf{0})$ is Newton non-degenerate (respectively, strongly Newton non-degenerate) over every compact face $\Delta(\subset \Gamma(f))$.

Remark 16 (cf. [3], Remark 4, [11], [10]). Let $f(\boldsymbol{z})$ be a holomorphic weighted homogeneous polynomial of positive degree with respect to a strictly positive weight vector $P$. Then we have $f=f_{\Delta(P)}$.

(i) Suppose that $f$ is Newton non-degenerate over $\Delta(P)$, namely, 0 is not a critical value of $f_{\Delta(P)}=f$ : $\mathbb{C}^{* n} \rightarrow \mathbb{C}$. Then we can show that $f_{\Delta(P)}=f: \mathbb{C}^{* n} \rightarrow \mathbb{C}$ has no critical point. Hence, using (ii) below, we see that $f$ is strongly Newton non-degenerate over $\Delta(P)$.

(ii) Suppose that $\operatorname{dim} \Delta(P) \geq 1$, namely, $f=f_{\Delta(P)}$ has at least two monomials. Then we can show that $f_{\Delta(P)}=f: \mathbb{C}^{* n} \rightarrow \mathbb{C}$ is surjective.

In the mixed cases, the situations are more complicated. Let $(f, \mathbf{0})$ be the germ of a mixed function $f$ at $\mathbf{0} \in \mathbb{C}^{n}$.

(iii) Suppose that $(f, \mathbf{0})$ is Newton non-degenerate over a compact face $\Delta=\Delta(P)(\subset \Gamma(f))$ and the face function $f_{P}$ is a mixed weighted homogeneous polynomial (Definition 10). Morerover, we assume $P \gg 0$ and $f_{P}$ is a polar weighted homogeneous polynomial of non-zero polar degree with respect to some weight vector $Q$. Then, we can show that $f_{P}: \mathbb{C}^{* n} \rightarrow \mathbb{C}$ has no mixed critical points.

(iv) In addition to (iii), we assume that $f_{P}^{-1}(0) \cap \mathbb{C}^{* n} \neq \emptyset$. Then we can show that $f_{P}: \mathbb{C}^{* n} \rightarrow \mathbb{C}$ is surjective. Hence, with tha fact (iii), in this case, Newton non-degeneracy over a compact face $\Delta(P)$ implies strong Newton non-degeneracy over $\Delta(P)$.

The proofs of (i) - (iv) are given in [11] and [10] in detail.

\footnotetext{
${ }^{3}$ In [4, a convenient (see Definition 25] of this paper) mixed function $f(\boldsymbol{z}, \overline{\boldsymbol{z}})$ is called of strongly polar positive (mixed) weighted homogeneous face type if the face function $f_{\Delta}(\boldsymbol{z}, \overline{\boldsymbol{z}})$ is a strongly polar positive (mixed) weighted homogeneous polynomial for every $(n-1)$-dimensional face. After this definition, Proposition 10 of 4 proves that for a convenient mixed function $f$ of strongly polar positive (mixed) weighted homogeneous face type (in the sense of [4]) and any weight vector $P$, the face function $f_{\Delta}(P)$ is also a strongly polar positive mixed weighted homogeneous polynomial with respect to $P$.

${ }^{4}$ In this case we do not need the surjectivity from $\mathbb{C}^{* n}$ onto $\mathbb{C}$.
} 


\section{Admissible REgular simplicial CONE SUbDivisions}

A regular simplicial cone subdivision of $N_{\mathbb{R}}^{+}$is defined as in [5], p.86, §5.6. Let us introduce the notions of convenient subdivisions. We define

$$
E_{j}:={ }^{t}(0, \ldots, 0,1,0, \ldots, 0)
$$

for $j(=1, \ldots, n)$. Namely, $\left\{E_{1}, \ldots, E_{n}\right\}$ is the canonical basis of $\mathbb{Z}^{n}$.

Definition $17\left([5]\right.$, p.90). We say a regular simplicial cone subdivision $\Sigma^{*}$ is convenient if $\Sigma^{*}$ contains all cones

$$
E_{I}:=\operatorname{Cone}\left(E_{i_{1}}, \ldots, E_{i_{k}}\right)
$$

with $I:=\left\{i_{1}, \ldots, i_{k}\right\}(\varsubsetneqq\{1, \ldots, n\})$. Thus $\Sigma^{*}$ contains Cone $\left(E_{1}\right), \ldots$, Cone $\left(E_{n}\right)$.

Let $\mathcal{V}$ be the set of all vertices of a regular simplicial cone subdivision $\Sigma^{*}$, and $\mathcal{V}^{+}$be the set of all strictly positive vertices of $\Sigma^{*}$.

Remark 18. For a convenient regular simplicial cone subdivision $\Sigma^{*}$, the following facts are important:

(1) The vertices except $E_{1}, \ldots, E_{n}$ of $\Sigma^{*}$ are all strictly positive 5 . Namely, $\mathcal{V} \backslash\left\{E_{1}, \ldots, E_{n}\right\} \subset \mathcal{V}^{+}$. Thus we have

$$
\mathcal{V}=\mathcal{V}^{+} \cup\left\{E_{1}, \ldots, E_{n}\right\}
$$

(2) The toric modification (see [1],p.73, [3] or [5],p.89 for the definition)

$$
\hat{\pi}: X \rightarrow \mathbb{C}^{n}
$$

associated with $\Sigma^{*}$ is a proper birational map and $\hat{\pi}: X \backslash \hat{\pi}^{-1}(\mathbf{0}) \rightarrow \mathbb{C}^{n} \backslash\{\mathbf{0}\}$ is biholomorphic and we have

$$
\hat{\pi}^{-1}(\mathbf{0})=\bigcup_{P \gg 0} \hat{E}(P),
$$

where $\hat{E}(P)$ is the exceptional divisor ([1], p.74) defined by a vertex $P$ of $\Sigma^{*}$. See [1, Theorem(1.4), Corollary(1.4.1), and also [5, Theorem 5.16.

Now for the germ $(f, \mathbf{0})$ of a mixed function $f$, we can also define the (radial) dual Newton diagram $\Gamma^{*}(f)$ of $(f, \mathbf{0})$ in the same way as holomorphic function germs at $\mathbf{0}$ (see [1],p.120, [5], p.97).

Definition 19 (admissible cone, [1],p.121). Let $\tau=\operatorname{Cone}\left(P_{1}, \ldots, P_{s}\right)$, where $P_{1}, \ldots, P_{s} \in N_{\mathbb{R}}^{+}$, be a cone in $N_{\mathbb{R}}^{+}$, that is,

$$
\tau:=\left\{\sum_{j=1}^{s} r_{j} P_{j} \mid r_{j} \geq 0, j=1, \ldots, s\right\} .
$$

We set

$$
\operatorname{Int}(\tau):=\left\{\sum_{j=1}^{s} r_{j} P_{j} \mid r_{1}>0, \ldots, r_{s}>0\right\},
$$

which we call the interior of $\tau$, and

$$
P_{\tau}:=P_{1}+\cdots+P_{s} .
$$

We say (cf. [1],p.121,Definition (3.1.3)) a cone $\tau$ is admissible for $\Gamma^{*}(f)$ if the interior $\operatorname{Int}(\tau)$ is a subset of an equivalence class in $\Gamma^{*}(f)$, that is, $\operatorname{Int}(\tau)$ is contained in the equivalence class $\left[P_{\tau}\right]$.

Definition 20 (admissible regular simplicial cone subdivision, 1],p.121, [5,p.98). Let $\Sigma^{*}$ be a regular simplicial cone subdivision of $N_{\mathbb{R}}^{+}$. We say that $\Sigma^{*}$ is admissible for the germ $(f, \mathbf{0})$ of a mixed function $f$ (or for $\Gamma^{*}(f)$ ) if every cone $\tau$ in $\Sigma^{*}$ is admissible for $\Gamma^{*}(f)$.

\footnotetext{
${ }^{5}$ The proof is as follows. Let $P(\neq \mathbf{0})$ be a vertex in $\mathcal{V} \backslash\left\{E_{1}, \ldots, E_{n}\right\}$. Suppose that $P$ is not strictly positive. Then we may assume that $P={ }^{t}\left(p_{1}, \ldots, p_{n}\right)$ with $p_{1}>0, \ldots, p_{s}>0$ and $p_{s+1}=\cdots=p_{n}=0$. Here we have $2 \leq s<n$. Since $\Sigma^{*}$ is convenient and $s<n$, we see $\operatorname{Cone}\left(E_{1}, \ldots, E_{s}\right) \in \Sigma^{*}$. We also see $\operatorname{Cone}(P) \neq \operatorname{Cone}\left(E_{1}, \ldots, E_{s}\right)$ as $s \geq 2$. Then, by the definition of simplicial cone subdivisions (see [5], p.86), we have $P \in \operatorname{Cone}(P) \cap \operatorname{Cone}\left(E_{1}, \ldots, E_{s}\right) \subset \partial\left(\operatorname{Cone}\left(E_{1}, \ldots, E_{s}\right)\right)$. This is a contradiction. Hence, $P$ is strictly positive.
} 
Proposition 21 ([1], p.121, Proposition (3.1.4)). Let $\tau=\operatorname{Cone}\left(P_{1}, \ldots, P_{s}\right)$, where $P_{1}, \ldots, P_{s} \in N_{\mathbb{R}}^{+}$, be a cone in $N_{\mathbb{R}}^{+}$. If $\tau$ is admissible for $\Gamma^{*}(f)$, then $\Delta\left(P_{i}\right) \supset \Delta\left(P_{\tau}\right)$ for any $i=1, \ldots, s$, where $P_{\tau}:=P_{1}+\cdots+P_{s}$. In particular, we have

$$
\bigcap_{i=1}^{s} \Delta\left(P_{i}\right) \supset \Delta\left(P_{\tau}\right)=\Delta(P)(\neq \emptyset)
$$

for every $P$ in $\operatorname{Int}(\tau)$, and therefore we have $\bigcap_{i=1}^{s} \Delta\left(P_{i}\right) \neq \emptyset$.

Proposition 22. Let $\tau=\operatorname{Cone}\left(P_{1}, \ldots, P_{s}\right)$, where $P_{1}, \ldots, P_{s} \in N_{\mathbb{R}}^{+}$, be a cone in $N_{\mathbb{R}}^{+}$. If $\tau$ is admissible for $\Gamma^{*}(f)$, then we have

$$
\bigcap_{i=1}^{s} \Delta\left(P_{i}\right)=\Delta(P)
$$

for every $P$ in $\operatorname{Int}(\tau)$.

Proof. By Proposition 21, we have $\bigcap_{i=1}^{s} \Delta\left(P_{i}\right) \supset \Delta\left(P_{\tau}\right) \neq \emptyset$, where $P_{\tau}:=P_{1}+\cdots+P_{s}$. Hence, take a point $\boldsymbol{x} \in \Delta\left(P_{\tau}\right)$. Then, since $\boldsymbol{x} \in \Delta\left(P_{i}\right)$ for any $i=1, \ldots, s$, we have

$$
P_{\tau}(\boldsymbol{x})=\sum_{i=1}^{s} P_{i}(\boldsymbol{x})=\sum_{i=1}^{s} d\left(P_{i}\right) .
$$

On the other hand, for any $\boldsymbol{y} \in \Gamma_{+}(f)$, we have

$$
P_{\tau}(\boldsymbol{y})=\sum_{i=1}^{s} P_{i}(\boldsymbol{y}) \geq \sum_{i=1}^{s} d\left(P_{i}\right) .
$$

Thus we conclude that

$$
d\left(P_{\tau}\right)=\sum_{i=1}^{s} d\left(P_{i}\right)
$$

Let $\boldsymbol{x}^{\prime}$ be an arbitrary point of $\bigcap_{i=1}^{s} \Delta\left(P_{i}\right)$. Then we have

$$
P_{\tau}\left(\boldsymbol{x}^{\prime}\right)=\sum_{i=1}^{s} P_{i}\left(\boldsymbol{x}^{\prime}\right)=\sum_{i=1}^{s} d\left(P_{i}\right)=d\left(P_{\tau}\right) .
$$

Thus we see that $\boldsymbol{x}^{\prime}$ is contained in $\Delta\left(P_{\tau}\right)$. Since $\operatorname{Int}(\tau)$ is a subset of an equivalence class in $\Gamma^{*}(f)$, we have $\Delta\left(P_{\tau}\right)=\Delta(P)$ for every $P$ in $\operatorname{Int}(\tau)$.

Proposition 23. Consider the germ $(f, \mathbf{0})$ of a mixed function $f$ and let $\Sigma^{*}$ be a regular simplicial cone subdivision which is admissible for $f$. Then, for every face $\Delta(P)$, there exist vertices $Q_{1}, \ldots, Q_{s}$ in $\Sigma^{*}$ such that

$$
\Delta(P) \subset \bigcap_{j=1}^{s} \Delta\left(Q_{j}\right) .
$$

Proof. Suppose that $\operatorname{dim} \Delta(P)=n-1$. There exist vertices $Q_{1}, \ldots, Q_{s}$ in $\Sigma^{*}$ such that $\Delta(P) \subset$ $\bigcap_{j=1}^{s} \Delta\left(Q_{j}\right)$, where, of course, we take $s=1$ and $Q_{1}=P$. Suppose that $\operatorname{dim} \Delta(P)=n-2$. Then, in the (radial) dual Newton diagram $\Gamma^{*}(f), P$ is contained in the interior of some admissible (for $\Gamma^{*}(f)$ ) cone $\Xi:=$ Cone $\left(Q_{1}, \ldots, Q_{s}\right)$ whose vetices $Q_{1}, \ldots, Q_{s}$ satisfy $\operatorname{dim} \Delta\left(Q_{j}\right)=n-1$ for every $j=1, \ldots, s$. Hence, $P=\sum_{j=1}^{s} a_{j} Q_{j}$ for some $a_{j}>0(j=1, \ldots, s)$. By Proposition 21, we have $\Delta(P) \subset \bigcap_{j=1}^{s} \Delta\left(Q_{j}\right)$. Here the weight vectors $Q_{1}, \ldots, Q_{s}$, multiplied by some real positive numbers if necessary, are vertices of $\Sigma^{*}$.

Suppose that $\operatorname{dim} \Delta(P)=k(k \leq n-3)$ and the assertion is true for faces with $\operatorname{dim} \Delta \geq k+1$. Then, in the (radial) dual Newton diagram $\Gamma^{*}(f), P$ is contained in the interior of some admissible (for $\Gamma^{*}(f)$ )

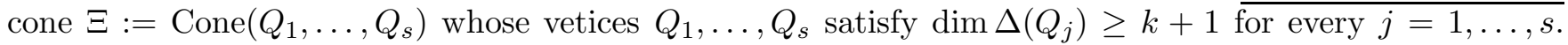
Hence, $P=\sum_{j=1}^{s} a_{j} Q_{j}$ for some $a_{j}>0(j=1, \ldots, s)$. By Proposition 21, we have $\Delta(P) \subset \bigcap_{j=1}^{s} \Delta\left(Q_{j}\right)$. 
Here, by the induction hypothesis, for each face $\Delta\left(Q_{j}\right)$, there exist vertices $Q_{j 1}^{\prime}, \ldots, Q_{j s_{j}}^{\prime}$ in $\Sigma^{*}$ such that $\Delta\left(Q_{j}\right) \subset \bigcap_{\ell=1}^{s_{j}} \Delta\left(Q_{j \ell}^{\prime}\right)$. Thus we have

$$
\Delta(P) \subset \bigcap_{j=1}^{s}\left(\bigcap_{\ell=1}^{s_{j}} \Delta\left(Q_{j \ell}^{\prime}\right)\right) .
$$

\section{CONVEniEnt MiXed FUnCTION GERMS}

Let $(f, \mathbf{0})$ be the germ of a mixed function $f$ at $\mathbf{0} \in \mathbb{C}^{n}$. For $I \subset\{1, \ldots, n\}, f^{I}$ denotes the restriction of $f$ on the coordinate subspace

$$
\mathbb{C}^{I}:=\left\{\boldsymbol{z} \mid z_{j}=0, j \notin I\right\} .
$$

Definition 24 ([5], p.98). Let $\Sigma^{*}$ be a regular simplicial cone subdivision which is admissible for $f$ (Definition 201). Then we say $\Sigma^{*}$ is convenient if for every $I$ with $f^{I} \not \equiv 0$, the cone $E_{I^{c}}$ is contained in $\Sigma^{*}$.

Definition 25 (convenient function germ, 5], p.79). The germ $(f, \mathbf{0})$ of a mixed function is called convenient if $f^{I} \not \equiv 0$ for every $I \neq \emptyset$.

Remark 26. The germ $(f, \mathbf{0})$ of a mixed function $f$ is convenient (Definition 25) if and only if $f$ has some terms $c_{\nu, \mu} z_{i}^{\nu_{i}} \bar{z}_{i}^{\mu_{i}}\left(c_{\nu, \mu} \neq 0\right)$, where $\nu=\left(0, \ldots, 0, \nu_{i}, 0, \ldots, 0\right), \mu=\left(0, \ldots, 0, \mu_{i}, 0, \ldots, 0\right)$, for every $i(=1, \ldots, n)$.

Remark 27. Suppose that (i) $\Sigma^{*}$ is a convenient (in the sense of Definition 24) regular simplicial cone subdivision which is admissible for $f$ and (ii) the germ of a mixed function $f$ is convenient (Definition 25). Then $\Sigma^{*}$ is convenient in the sense of Definition 17. Hence, for the toric modification $\hat{\pi}: X \rightarrow \mathbb{C}^{n}$ associated with $\Sigma^{*}$, the restriction $\hat{\pi}: X \backslash \hat{\pi}^{-1}(\mathbf{0}) \rightarrow \mathbb{C}^{n} \backslash\{\mathbf{0}\}$ is biholomorphic and we have $\hat{\pi}^{-1}(\mathbf{0})=\bigcup_{P \gg 0} \hat{E}(P)$ (recall Remark 18).

Theorem 28 (Isolatedness, [3], Theorem $19(1))$. Let $(f, \mathbf{0})$ be a convenient Newton non-degenerate 6 mixed function germ at $\mathbf{0} \in \mathbb{C}^{n}$. Then $\mathbf{0}$ is a mixed regular point of $f$ or an isolated singular point (Definition 1) of the mixed hypersurface $V:=f^{-1}(0)$.

Lemma 29. Suppose that $n \geq 2$, the germ $(f, \mathbf{0})$ of a mixed function $f(\boldsymbol{z}, \overline{\boldsymbol{z}})=\sum_{\nu, \mu} c_{\nu, \mu} \boldsymbol{z}^{\nu} \overline{\boldsymbol{z}}^{\mu}$ is convenient (Definition 25) and $\Sigma^{*}$ is a convenient (in the sense of Definition 24) regular simplicial cone subdivision which is admissible for $f$. Then we have the following:

(1) All the 1-dimensional cones $\operatorname{Cone}(P)$ with non-strictly positive vertex $P$ in the (radial) dual Newton diagram $\Gamma^{*}(f)$ are Cone $\left(E_{1}\right), \ldots$, and Cone $\left(E_{n}\right)$.

(2) For a fixed $j(=1, \ldots, n)$, consider the face $\Delta\left(E_{j}\right)^{7}$. If $c_{\nu, \mu} \neq 0$ and $\nu+\mu \in \Delta\left(E_{j}\right)$, then we see that $E_{j}(\nu+\mu)=0$ and $E_{j}(\nu-\mu)=0$.

Proof. If $n \geq 2$, the germ $(f, \mathbf{0})$ of a mixed function $f$ is convenient and $\Sigma^{*}$ is a convenient (in the sense of Definition 24) regular simplicial cone subdivision which is admissible for $f$, then, as stated in Remark 27, $\Sigma^{*}$ is convenient in the sense of Definition 17. Hence, by Remark 18, all the 1-dimensional cones Cone $(P)$ with non-strictly positive vertex $P$ in the (radial) dual Newton diagram $\Gamma^{*}(f)$ are $\operatorname{Cone}\left(E_{1}\right), \ldots$, Cone $\left(E_{n}\right)$. By Remark 26, $f$ has some terms $c_{\nu, \mu} z_{i}^{\nu_{i}} \bar{z}_{i}^{\mu_{i}}\left(c_{\nu, \mu} \neq 0\right)$, where $\nu=\left(0, \ldots, 0, \nu_{i}, 0, \ldots, 0\right), \mu=\left(0, \ldots, 0, \mu_{i}, 0, \ldots, 0\right)$, for every $i(=1, \ldots, n)$. For the weight vector $E_{j}$ and the term $c_{\nu, \mu} z_{i}^{\nu_{i}} \bar{z}_{i}^{\mu_{i}}$ with $i \neq j$ and $c_{\nu, \mu} \neq 0$, we have

$$
E_{j}(\nu+\mu)=\nu_{j}+\mu_{j}=0 .
$$

Hence, we see that the minimum value $d\left(E_{j}\right)$ is equal to 0 . Thus, we have

$$
\Delta\left(E_{j}\right)=\left\{\xi \in \Gamma_{+}(f) \mid E_{j}(\xi)=0\right\}
$$

\footnotetext{
${ }^{6}$ In Theorem 19 of 3 , the assertion (1) holds without "true non-degeneracy" assumption.

${ }^{7} \Delta\left(E_{j}\right)$ is not compact.
} 
for every $j(=1, \ldots, n)$. Thus, if $c_{\nu, \mu} \neq 0$ and $\nu+\mu \in \Delta\left(E_{j}\right)$, then we have

$$
E_{j}(\nu+\mu)=\nu_{j}+\mu_{j}=0
$$

and hence, we have

$$
\nu_{j}=\mu_{j}=0
$$

Thus, moreover, we have

$$
E_{j}(\nu-\mu)=\nu_{j}-\mu_{j}=0
$$

We have an analogy of Proposition 10 (4]) for mixed function germs of strongly polar non-negative mixed weighted homogeneous face type:

Proposition 30. Suppose that (1) $n \geq 2$, (2) the germ $(f, \mathbf{0})$ of a mixed function $f(\boldsymbol{z}, \overline{\boldsymbol{z}})=\sum_{\nu, \mu} c_{\nu, \mu} \boldsymbol{z}^{\nu} \overline{\boldsymbol{z}}^{\mu}$ is convenient, and (3) $\Sigma^{*}$ is a convenient (Definition 24) regular simplicial cone subdivision which is admissible for $f$. If the germ $(f, \mathbf{0})$ of a mixed function $f$ is of strongly polar non-negative mixed weighted homogeneous face type (Definition 12), then for any strictly positive weight vector $P, f_{P}$ is a strongly polar non-negative mixed weighted homogeneous polynomial with respect to the weight vector $P$.

Proof. By the definition, for every compact face $\Delta$, the face function $f_{\Delta}(\boldsymbol{z}, \overline{\boldsymbol{z}})$ is a strongly polar nonnegative mixed weighted homogeneous polynomial (Definition 11).

Suppose that $\operatorname{dim} \Delta(P)=n-1$. By the assumption, $f_{P}$ is a strongly polar non-negative mixed weighted homogeneous polynomial with respect to $P$ since $P$ is unique up to multiplications of positive real numbers. Suppose that $\operatorname{dim} \Delta(P)=n-2$. Then, in the (radial) dual Newton diagram $\Gamma^{*}(f), P$ is contained in the interior of some admissible (for $\Gamma^{*}(f)$ ) cone $\Xi:=$ Cone $\left(Q_{1}, \ldots, Q_{s}\right.$ ) whose vetices $Q_{1}, \ldots, Q_{s}$ satisfy $\operatorname{dim} \Delta\left(Q_{j}\right)=n-1$ for every $j=1, \ldots, s$. We have $P=\sum_{j=1}^{s} a_{j} Q_{j}$ for some $a_{j}>0(j=1, \ldots, s)$. By Proposition 21, we have

$$
\Delta(P) \subset \bigcap_{j=1}^{s} \Delta\left(Q_{j}\right) .
$$

Here the weight vectors $Q_{1}, \ldots, Q_{s}$, multiplied by some real positive numbers if necessary, are vertices of $\Sigma^{*}$. If $c_{\nu, \mu} \neq 0$ and $\nu+\mu \in \Delta(P)$, then we have $\nu+\mu \in \Delta\left(Q_{j}\right)$ for every $j=1, \ldots, s$. By the assumptions of this Proposition, all the non-strictly positive vertices of $\Sigma^{*}$ are $E_{1}, \ldots, E_{n}$. If $c_{\nu, \mu} \neq 0$ and $\nu+\mu \in \Delta\left(E_{j}\right)$, then, by Lemma 29, we have $E_{j}(\nu+\mu)=0$ and $E_{j}(\nu-\mu)=0$. Hence, we may say that for every $(\nu, \mu)$ with $c_{\nu, \mu} \neq 0$ and $\nu+\mu \in \Delta(P)$,

$$
P(\nu-\mu)=\sum_{j=1}^{s} a_{j} Q_{j}(\nu-\mu)=\sum_{j=1}^{t} a_{j} Q_{j}(\nu-\mu),
$$

where $Q_{1}, \ldots, Q_{t}$ are all strictly positive. By the above result on $(n-1)$-dimensional case, the numbers $Q_{1}(\nu-\mu), \ldots, Q_{t}(\nu-\mu)$ are all non-negative, and each number $m_{j}:=Q_{j}(\nu-\mu)$ does not depend on $(\nu, \mu)$ with $c_{\nu, \mu} \neq 0$ and $\nu+\mu \in \Delta(P)$. Thus we conclude that

$$
\operatorname{pdeg}_{P} f_{P}=P(\nu-\mu)=\sum_{j=1}^{t} a_{j} m_{j} \geq 0 .
$$

Now suppose that $\operatorname{dim} \Delta(P)=k(k \leq n-3)$ and the assertion is true for faces with $\operatorname{dim} \Delta \geq k+1$. In the (radial) dual Newton diagram $\Gamma^{*}(f), P$ is contained in the interior of some admissible (for $\Gamma^{*}(f)$ ) cone $\Xi:=$ Cone $\left(Q_{1}, \ldots, Q_{s}\right)$ whose vetices $Q_{1}, \ldots, Q_{s}$ satisfy $\operatorname{dim} \Delta\left(Q_{j}\right) \geq k+1$ for every $j=1, \ldots, s$. We have $P=\sum_{j=1}^{s} a_{j} Q_{j}$ for some $a_{j}>0(j=1, \ldots, s)$. By Proposition 21, we have $\Delta(P) \subset \bigcap_{j=1}^{s} \Delta\left(Q_{j}\right)$. Here, by using Proposition [23, we may say that all $Q_{1}, \ldots, Q_{s}$ are vetices of $\Sigma^{*}$. By the assumptions of this Proposition, all the non-strictly positive vertices of $\Sigma^{*}$ are $E_{1}, \ldots, E_{n}$. If $c_{\nu, \mu} \neq 0$ and $\nu+\mu \in \Delta\left(E_{j}\right)$, 
then, by Lemma 29, we have $E_{j}(\nu+\mu)=0$ and $E_{j}(\nu-\mu)=0$. Hence, we may say that for every $(\nu, \mu)$ with $c_{\nu, \mu} \neq 0$ and $\nu+\mu \in \Delta(P)$,

$$
P(\nu-\mu)=\sum_{j=1}^{s} a_{j} Q_{j}(\nu-\mu)=\sum_{j=1}^{t} a_{j} Q_{j}(\nu-\mu)
$$

where $Q_{1}, \ldots, Q_{t}$ are all strictly positive. By the induction hypothesis, the numbers $Q_{1}(\nu-\mu), \ldots, Q_{t}(\nu-\mu)$ are all non-negative, and each number $m_{j}:=Q_{j}(\nu-\mu)$ does not depend on $(\nu, \mu)$ with $c_{\nu, \mu} \neq 0$ and $\nu+\mu \in \Delta(P)$. Thus we see that

$$
\operatorname{pdeg}_{P} f_{P}=P(\nu-\mu)=\sum_{j=1}^{t} a_{j} m_{j} \geq 0
$$

\section{Resolutions of NeWton NON-DEgenerate miXed Polynomials of STRONGly polar NON-NEGATIVE MIXED WEIGHTED HOMOGENEOUS FACE TYPE}

Theorem 11 ([4]) and Theorem 9.19 ([5]) are generalizations of Varchenko's famous Lemma 10.3 ([12]) on holomorphic function germs to mixed polynomial germs or mixed function germs.

Our main Theorem 32 below improves Theorem $11(1),(2)$ of 4 under the Assumption $(*)$. In order to prove Theorem 32, we prepare the following Lemma 31.

Here we say a complex valued function $\rho(\boldsymbol{u})$ with complex $n$ variables $\boldsymbol{u}=\left(u_{1}, \ldots, u_{n}\right), u_{j}=x_{j}+i y_{j}(j=$ $1, \ldots, n)$ is of class $C^{s}$ if the real and imaginary parts of $\rho(\boldsymbol{u})=\rho\left(x_{1}, y_{1}, \ldots, x_{n}, y_{n}\right)$ are $C^{s}$-functions with real $2 n$ variables.

If the real and imaginary parts of $\rho(\boldsymbol{u})=\rho\left(x_{1}, y_{1}, \ldots, x_{n}, y_{n}\right)$ are of class $C^{1}$, namely, they have their

partial derivatives $\frac{\partial \Re \rho}{\partial x_{j}}, \frac{\partial \Re \rho}{\partial y_{j}}, \frac{\partial \Im \rho}{\partial x_{j}}, \frac{\partial \Im \rho}{\partial y_{j}}$ and these 4 functions are continuous, then by (2.1), $\frac{\partial \rho}{\partial z_{j}}$ and $\frac{\partial \rho}{\partial \bar{z}_{j}}$ exist and they are continuous. Conversely, if $\frac{\partial \rho}{\partial z_{j}}$ and $\frac{\partial \rho}{\partial \bar{z}_{j}}$ exist and they are continuous, then by (2.2), the real and imaginary parts of $\rho(\boldsymbol{u})=\rho\left(x_{1}, y_{1}, \ldots, x_{n}, y_{n}\right)$ are of class $C^{1}$.

Lemma 31. Let $r, s(\geq 1)$ be integers, and $u$ be a complex variable. We set

$$
\xi(u):= \begin{cases}\frac{u^{r+s}}{\bar{u}^{r}} & (u \neq 0) \\ 0 & (u=0)\end{cases}
$$

Then $\xi(u)$ is of class $C^{s-1}$ on $\mathbb{C}$. Hence,

$$
\bar{\xi}(u)= \begin{cases}\frac{\bar{u}^{r+s}}{u^{r}} & (u \neq 0) \\ 0 & (u=0)\end{cases}
$$

is also of class $C^{s-1}$ on $\mathbb{C}$.

Proof. We have

$$
\xi(u)=\xi(x, y)=\frac{u^{r+s}}{\bar{u}^{r}}=\frac{u^{r+s+r}}{|u|^{2 r}}=\frac{(x+i y)^{2 r+s}}{{\sqrt{x^{2}+y^{2}}}^{2 r}}=\frac{(x+i y)^{2 r+s}}{\left(x^{2}+y^{2}\right)^{r}} \quad(\text { if }(x, y) \neq(0,0)) .
$$

The real and imaginary parts of the last numerator of (8.1) are of degree $2 r+s$. We will prove that the real and imaginary parts of $\xi(u)=\xi(x, y)$ are of class $C^{s-1}$. To do this, it is sufficient that we show

$$
\varphi(x, y):= \begin{cases}\frac{x^{2 r+s-j} y^{j}}{\left(x^{2}+y^{2}\right)^{r}} & ((x, y) \neq(0,0)) \\ 0 & ((x, y)=(0,0))\end{cases}
$$


is a $C^{s-1}$-function on $\mathbb{R}^{2}$ for every $r(\geq 1)$ and every $j(0 \leq j \leq 2 r+s)$. Therefore we will prove this assertion by the induction on $s$.

If $s=1$, then

$$
|\varphi(x, y)|=\frac{|x|^{2 r+1-j}|y|^{j}}{{\sqrt{x^{2}+y^{2}}}^{2 r}} \leq|x| \text { or }|y| \quad(\text { if }(x, y) \neq(0,0)) \text {. }
$$

Hence, $\varphi(x, y)$ is a continuous, namely, $C^{0}$-function on $\mathbb{R}^{2}$. Thus the assertion is true for $s=1$.

Suppose that the assertion is true for $s$ (with $s \geq 1)$, every $r(\geq 1)$ and every $j(0 \leq j \leq 2 r+s)$. We will prove that

$$
\psi(x, y):= \begin{cases}\frac{x^{2 r+s+1-j} y^{j}}{\left(x^{2}+y^{2}\right)^{r}} & ((x, y) \neq(0,0)) \\ 0 & ((x, y)=(0,0))\end{cases}
$$

is a $C^{s}$-function on $\mathbb{R}^{2}$ for every $r(\geq 1)$ and $j(0 \leq j \leq 2 r+s+1)$.

We have

$$
\begin{aligned}
\frac{\partial \psi}{\partial x}(x, y) & =\frac{(2 r+s+1-j) x^{2 r+s-j} y^{j}\left(x^{2}+y^{2}\right)^{r}-2 r x \cdot x^{2 r+s+1-j} y^{j}\left(x^{2}+y^{2}\right)^{r-1}}{\left(x^{2}+y^{2}\right)^{2 r}} \\
& =\frac{(2 r+s+1-j) x^{2 r+s-j} y^{j}}{\left(x^{2}+y^{2}\right)^{r}}-2 r \frac{x^{2(r+1)+s-j} y^{j}}{\left(x^{2}+y^{2}\right)^{r+1}} \quad((x, y) \neq(0,0))
\end{aligned}
$$

and

Since

$$
\begin{aligned}
\frac{\partial \psi}{\partial y}(x, y) & =\frac{j x^{2 r+s+1-j} y^{j-1}\left(x^{2}+y^{2}\right)^{r}-2 r y \cdot x^{2 r+s+1-j} y^{j}\left(x^{2}+y^{2}\right)^{r-1}}{\left(x^{2}+y^{2}\right)^{2 r}} \\
& =\frac{j x^{2 r+s-(j-1)} y^{j-1}}{\left(x^{2}+y^{2}\right)^{r}}-2 r \frac{x^{2(r+1)+s-(j+1)} y^{j+1}}{\left(x^{2}+y^{2}\right)^{r+1}}((x, y) \neq(0,0)) .
\end{aligned}
$$

and

$$
\psi(x, 0)=x^{s+1-j} y^{j}= \begin{cases}x^{s+1} & (j=0) \\ 0 & (j \geq 1)\end{cases}
$$

we have

$$
\psi(0, y)=x^{2 r+s+1-j} y^{j-2 r}= \begin{cases}0 & (j \leq 2 r+s) \\ y^{s+1} & (j=2 r+s+1)\end{cases}
$$

$$
\frac{\partial \psi}{\partial x}(0,0)=\frac{\partial \psi}{\partial y}(0,0)=0 .
$$

Thus, by the induction hypothesis, $\frac{\partial \psi}{\partial x}(x, y)$ and $\frac{\partial \psi}{\partial y}(x, y)$ are $C^{s-1}$-functions on $\mathbb{R}^{2}$. Hence, $\psi(x, y)$ is a $C^{s}$-function on $\mathbb{R}^{2}$. This completes the proof of Lemma 31 .

We can give another proof using Wirtinger derivatives $\frac{\partial}{\partial u}, \frac{\partial}{\partial \bar{u}}$. We prove the assertion of Lemma 31 by the induction on $s$. If $s=1$, then $\xi(u)$ is a real analytic map on $\mathbb{C} \backslash\{0\}$. Since $|\xi(u)|=|u|$ (if $u \neq 0$ ), we have $\lim _{u \rightarrow 0} \xi(u)=0=\xi(0)$. Hence, $\xi(u)$ is a continuous, namely, $C^{0}$-map on $\mathbb{C}$. Thus the assertion is true for $s=1$. Suppose that the assertion is true for $s$ (with $s \geq 1$ ) and every $r(\geq 1$ ). We will prove

$$
\eta(u):= \begin{cases}\frac{u^{r+s+1}}{\bar{u}^{r}} & (u \neq 0) \\ 0 & (u=0)\end{cases}
$$

is of class $C^{s}$ on $\mathbb{C}$ for every $r(\geq 1)$. We have

$$
\frac{\partial \eta}{\partial u}(u)=\frac{(r+s+1) u^{r+s} \bar{u}^{r}-u^{r+s+1} \cdot 0}{\bar{u}^{2 r}}=(r+s+1) \frac{u^{r+s}}{\bar{u}^{r}} \quad(\text { if } u \neq 0)
$$

and

$$
\frac{\partial \eta}{\partial \bar{u}}(u)=\frac{0 \cdot \bar{u}^{r}-r u^{r+s+1} \bar{u}^{r-1}}{\bar{u}^{2 r}}=-r \frac{u^{r+1+s}}{\bar{u}^{r+1}} \quad(\text { if } u \neq 0) .
$$

We set $u=x+i y=(x, y)(x, y \in \mathbb{R})$. Since $\eta(x, 0)=x^{s+1}$ and $\eta(0, y)=(i y)^{r+s+1}(-i y)^{-r}=(-1)^{r} i^{s+1} y^{s+1}$, we have

$$
\frac{\partial \eta}{\partial x}(0,0)=0 \text { and } \frac{\partial \eta}{\partial y}(0,0)=0 .
$$


Hence, we have

$$
\frac{\partial \eta}{\partial u}(0)=0
$$

and

$$
\frac{\partial \eta}{\partial \bar{u}}(0)=0
$$

From (8.2) and (8.4), by the induction hypothesis, we see $\frac{\partial \eta}{\partial u}$ is of class $C^{s-1}$ on $\mathbb{C}$. From (8.3) and (8.5), we also see $\frac{\partial \eta}{\partial \bar{u}}(u)$ is of class $C^{s-1}$ on $\mathbb{C}$. Hence, by (2.2),$\frac{\partial \eta}{\partial x}$ and $\frac{\partial \eta}{\partial y}$ are also of class $C^{s-1}$ on $\mathbb{C}$. Thus it is proved that $\eta$ is of class $C^{s}$ on $\mathbb{C}$. This completes the proof of Lemma 31 .

We now set up the situation of Theorem 32. Let $(f, \mathbf{0})$ be a convenient Newton non-degenerate mixed polynomial germ

$$
f(\boldsymbol{z}, \overline{\boldsymbol{z}})=\sum_{\nu, \mu} c_{\nu, \mu} \boldsymbol{z}^{\nu} \overline{\boldsymbol{z}}^{\mu}
$$

at $\mathbf{0} \in \mathbb{C}^{n}$ of strongly polar non-negative mixed weighted homogeneous face type (Definition 12). We assume $f(\mathbf{0})=0$. Then by Theorem [28, $\mathbf{0}$ is a mixed regular point of $f$ or an isolated singular point (Definition 10 of the mixed hypersurface $V:=f^{-1}(0)$.

Let $\Sigma^{*}$ be a regular simplicial subdivision (of $N_{\mathbb{R}}^{+}$) which is admissible for $f$ and assume that $\Sigma^{*}$ is convenient in the sense of Definition 24 (recall Remark 27). Consider the toric modification $\hat{\pi}: X \rightarrow \mathbb{C}^{n}$ associated with $\Sigma^{*}$. Then (recall Remark 18) the toric modification

$$
\hat{\pi}: X \rightarrow \mathbb{C}^{n}
$$

associated with $\Sigma^{*}$ is a proper birational map and $\hat{\pi}: X \backslash \hat{\pi}^{-1}(\mathbf{0}) \rightarrow \mathbb{C}^{n} \backslash\{\mathbf{0}\}$ is biholomorphic and we have

$$
\hat{\pi}^{-1}(\mathbf{0})=\bigcup_{P \gg 0} \hat{E}(P),
$$

where $\hat{E}(P)$ is the exceptional divisor defined by a vertex $P$ of $\Sigma^{*}$.

Let $(V, \mathbf{0})$ be the germ of the mixed hypersurface $V:=f^{-1}(0)$ at $\mathbf{0} \in \mathbb{C}^{n}$. We call

$$
\tilde{V}:=\text { the closure of } \hat{\pi}^{-1}(V \backslash\{\mathbf{0}\}) \text { in } X
$$

the strict transform of $V$ to $X$. We consider the strict transform $\tilde{V}$ as the germ at $\tilde{V} \cap \hat{\pi}^{-1}(\mathbf{0})$. For each $k$-dimensional cone $\tau=\operatorname{Cone}\left(P_{1}, \ldots, P_{k}\right)$ of $\Sigma^{*}$, we set

$$
\hat{E}(\tau)^{*}:=\left(\cap_{i=1}^{k} \hat{E}\left(P_{i}\right)\right) \backslash\left(\cup_{Q \in \mathcal{V}, Q \notin \tau} \hat{E}(Q)\right)
$$

and

$$
\tilde{V}(\tau)^{*}:=\tilde{V} \cap \hat{E}(\tau)^{*} .
$$

If the vertices of $\tau=\operatorname{Cone}\left(P_{1}, \ldots, P_{k}\right)$ of $\Sigma^{*}$ are all strictly positive and

$$
\left\{((\nu, \mu), j) \mid c_{\nu, \mu} \neq 0, \nu+\mu \notin \Delta\left(P_{j}\right), j=1, \ldots, k\right\} \neq \emptyset,
$$

where $r_{j}:=\operatorname{rdeg}_{P_{j}} f_{P_{j}}=d\left(P_{j}\right)$, then we define

$$
\Lambda(\tau):=\min \left\{P_{j}(\nu+\mu)-r_{j} \mid c_{\nu, \mu} \neq 0, \nu+\mu \notin \Delta\left(P_{j}\right), j=1, \ldots, k\right\} .
$$

By the definition, we have $\Lambda(\tau) \geq 1$. Moreover, we define $L\left(\Sigma^{*}\right)$ to be the set of all $\Lambda(\tau)$ 's such that

(1) $\tau$ is a cone of $\Sigma^{*}$ whose vertices are all strictly positive,

(2) $\left\{((\nu, \mu), j) \mid c_{\nu, \mu} \neq 0, \nu+\mu \notin \Delta\left(P_{j}\right), j=1, \ldots, k\right\} \neq \emptyset$, where $k:=\operatorname{dim} \tau$, and

(3) $\tilde{V}(\tau)^{*} \cap \mathbb{C}_{\sigma}^{n} \neq \emptyset$ for some $n$-dimensional cone $\sigma$ of $\Sigma^{*}$ with $\tau \prec \sigma$.

If $L\left(\Sigma^{*}\right) \neq \emptyset$, then we define

$$
\mathcal{L}:=\min L\left(\Sigma^{*}\right) .
$$

By the definition, we have $\mathcal{L} \geq 1$. 
Theorem 32. We assume $n \geq 2$. Under the situation mentioned above, we have the following:

(i) If the vertices of the $k$-dimensional cone $\tau$ of $\Sigma^{*}$ are all strictly positive, then $\tilde{V}(\tau)^{*}$ is empty or a real analytic manifold of dimension $2(n-k-1)$.

(ii) We assume:

Assumption $\left(^{*}\right)$ : For every point $\hat{\boldsymbol{u}}^{0} \in \tilde{V} \cap \hat{\pi}^{-1}(\mathbf{0})=\tilde{V} \cap \bigcup_{P \gg 0} \hat{E}(P)$, there exists an $n$-dimensional cone $\sigma=\left(P_{1}, \ldots, P_{n}\right)$ with strictly positive vertices $P_{1}, \ldots, P_{\kappa}(1 \leq \kappa \leq n)$ for which the toric chart $\mathbb{C}_{\sigma}^{n}$ contains $\hat{\boldsymbol{u}}^{0}$ and $\hat{\boldsymbol{u}}^{0}=\boldsymbol{u}_{\sigma}^{0}=(\underbrace{0, \ldots, 0}_{\kappa}, u_{\kappa+1}^{0}, \ldots, u_{n}^{0}), \quad u_{\kappa+1}^{0} \neq 0, \ldots, u_{n}^{0} \neq 0 \quad$ in $\mathbb{C}_{\sigma}^{n}$.

Then the strict transform germ $\tilde{V}$ at $\tilde{V} \cap \hat{\pi}^{-1}(\mathbf{0})$ is a $C^{\mathcal{L}-1}$-manifold if $L\left(\Sigma^{*}\right) \neq \emptyset$, and a real analytic manifold if $L\left(\Sigma^{*}\right)=\emptyset$. Hence, it is a topological manifold. Moreover, it is a real analytic manifold outside of $\tilde{V} \cap \hat{\pi}^{-1}(\mathbf{0})$.

Proof. We first prove the assertion (i). Let $\tau:=\left(P_{1}, \ldots, P_{k}\right)$ be a $k$-dimensional cone in $\Sigma^{*}$ whose vertices are all strictly positive vectors. Then the faces $\Delta\left(P_{1}\right), \ldots, \Delta\left(P_{k}\right)$ of $\Gamma_{+}(f)$ are all compact. If $\tilde{V}(\tau)^{*}=\emptyset$, then the proof is completed, and if $\tilde{V}(\tau)^{*} \neq \emptyset$, then we can take an $n$-dimensional cone

$$
\sigma=\left(P_{1}, \ldots, P_{n}\right)
$$

of $\Sigma^{*}$ and let

$$
\boldsymbol{u}_{\sigma}=\left(u_{\sigma 1}, \ldots, u_{\sigma n}\right)
$$

be the toric coordinates of the chart $\mathbb{C}_{\sigma}^{n}$. We write $u_{\sigma j}=u_{j}$ hereafter.

We put

$$
\Delta:=\bigcap_{j=1}^{k} \Delta\left(P_{j}\right)
$$

Then $\Delta$ is a compact face of $\Gamma_{+}(f)$ (recall Proposition 22$]^{8}$ ). For each $j=1, \ldots, k$, we set

$$
r_{j}:=\operatorname{rdeg}_{P_{j}} f_{P_{j}}, \quad \text { and } \quad p_{j}:=\operatorname{pdeg}_{P_{j}} f_{P_{j}} .
$$

By the strongly polar non-negative mixed weightedness assumption and Proposition 30, we have $r_{j}>0$ and $p_{j} \geq 0$ for every $j(=1, \ldots, k)$.

Put

$$
P_{j}={ }^{t}\left(p_{1 j}, \ldots, p_{n j}\right) .
$$

If $\nu+\mu \in \Delta\left(P_{j}\right)$, then we have

$$
\sum_{i=1}^{n} p_{i j}\left(\nu_{j}+\mu_{j}\right)=r_{j}>0 \quad \text { and } \quad \sum_{i=1}^{n} p_{i j}\left(\nu_{i}-\mu_{i}\right)=p_{j} \geq 0 .
$$

Hence, note that $r_{j}+p_{j}>0$ and $r_{j}+p_{j}=2 \sum_{i=1}^{n} p_{i j} \nu_{j}$ is an even positive integer. On the other hand, we have $r_{j}-p_{j}=2 \sum_{i=1}^{n} p_{i j} \mu_{j}$ is an even non-negative integer. Hence, we have two integers

$$
\frac{r_{j}+p_{j}}{2}>0, \quad \frac{r_{j}-p_{j}}{2} \geq 0 .
$$

Recall that $\hat{\pi}$ coincides with the toric morphism $\pi_{\sigma}$ on the toric chart $\mathbb{C}_{\sigma}^{n}$. Let us consider the pull-back polynomial

$$
\pi_{\sigma}^{*} f:=f \circ \pi_{\sigma}
$$

\footnotetext{
${ }^{8} \Delta=\Delta\left(P_{\tau}\right)$ where we set $P_{\tau}:=P_{1}+\cdots+P_{k}$. Hence, $P_{\tau}$ is a strictly positive vector.
} 
We decompose the polynomial $\pi_{\sigma}^{*} f$ "formally" as below:

$$
\begin{aligned}
& \pi_{\sigma}^{*} f(\boldsymbol{u}, \overline{\boldsymbol{u}}) \\
= & \sum_{\nu, \mu}^{P_{\nu}} c_{\nu} u_{1}^{P_{1}(\nu)} \cdots u_{n}^{P_{n}(\nu)} \bar{u}_{1}^{P_{1}(\mu)} \cdots \bar{u}_{n}^{P_{n}(\mu)} \\
= & \prod_{j=1}^{k} u_{j}^{\frac{r_{j}+p_{j}}{2}} \bar{u}_{j}^{\frac{r_{j}-p_{j}}{2}} \times\left(\sum_{\nu, \mu} c_{\nu, \mu} \prod_{j=1}^{k} u_{j}^{P_{j}(\nu)-\frac{r_{j}+p_{j}}{2}} \prod_{j=1}^{k} \bar{u}_{j}^{P_{j}(\mu)-\frac{r_{j}-p_{j}}{2}} u_{k+1}^{P_{k+1}(\nu)} \cdots u_{n}^{P_{n}(\nu)} \bar{u}_{k+1}^{P_{k+1}(\mu)} \cdots \bar{u}_{n}^{P_{n}(\mu)}\right),
\end{aligned}
$$

and we set

$$
\widetilde{f}(\boldsymbol{u}, \overline{\boldsymbol{u}}):=\sum_{\nu, \mu} c_{\nu, \mu} \prod_{j=1}^{k} u_{j}^{P_{j}(\nu)-\frac{r_{j}+p_{j}}{2}} \prod_{j=1}^{k} \bar{u}_{j}^{P_{j}(\mu)-\frac{r_{j}-p_{j}}{2}} u_{k+1}^{P_{k+1}(\nu)} \cdots u_{n}^{P_{n}(\nu)} \bar{u}_{k+1}^{P_{k+1}(\mu)} \cdots \bar{u}_{n}^{P_{n}(\mu)} .
$$

For every $j(=1, \ldots, k)$, we have

$$
u_{j}^{P_{j}(\nu)} \bar{u}_{j}^{P_{j}(\mu)}=u_{j}^{P_{j}(\nu+\mu) / 2+P_{j}(\nu-\mu) / 2} \bar{u}_{j}^{P_{j}(\nu+\mu) / 2-P_{j}(\nu-\mu) / 2} .
$$

If $\nu+\mu \in \Delta$, then we have

$$
u_{j}^{P_{j}(\nu)} \bar{u}_{j}^{P_{j}(\mu)}=u_{j}^{\frac{r_{j}+p_{j}}{2}} \bar{u}_{j}^{\frac{r_{j}-p_{j}}{2}}
$$

for every $j(=1, \ldots, k)$ by the strongly mixed weightedness assumption. Put

$$
\begin{aligned}
\widetilde{f}_{\Delta} & :=\sum_{\nu+\mu \in \Delta} c_{\nu, \mu} \prod_{j=1}^{k} u_{j}^{P_{j}(\nu)-\frac{r_{j}+p_{j}}{2}} \prod_{j=1}^{k} \bar{u}_{j}^{P_{j}(\mu)-\frac{r_{j}-p_{j}}{2}} u_{k+1}^{P_{k+1}(\nu)} \cdots u_{n}^{P_{n}(\nu)} \bar{u}_{k+1}^{P_{k+1}(\mu)} \cdots \bar{u}_{n}^{P_{n}(\mu)} \\
& =\sum_{\nu+\mu \in \Delta} c_{\nu, \mu} u_{k+1}^{P_{k+1}(\nu)} \cdots u_{n}^{P_{n}(\nu)} \bar{u}_{k+1}^{P_{k+1}(\mu)} \cdots \bar{u}_{n}^{P_{n}(\mu)} .
\end{aligned}
$$

Note that $\widetilde{f}_{\Delta}$ is a mixed polynomial having only the variables $u_{k+1}, \ldots, u_{n}$, namely, $\widetilde{f}_{\Delta}$ does not contain any variables $u_{1}, \ldots, u_{k}$ ).

Thus we obtain the following "formal" expression:

$$
\widetilde{f}(\boldsymbol{u}, \overline{\boldsymbol{u}})=\widetilde{f}_{\Delta}\left(\boldsymbol{u}^{\prime}, \overline{\boldsymbol{u}}^{\prime}\right)+\widetilde{R}(\boldsymbol{u}, \overline{\boldsymbol{u}})
$$

where $\boldsymbol{u}^{\prime}:=\left(u_{k+1}, \ldots, u_{n}\right)$ and

$$
\widetilde{R}(\boldsymbol{u}, \overline{\boldsymbol{u}}):=\sum_{\nu+\mu \notin \Delta} c_{\nu, \mu} \prod_{j=1}^{k} u_{j}^{P_{j}(\nu)-\frac{r_{j}+p_{j}}{2}} \prod_{j=1}^{k} \bar{u}_{j}^{P_{j}(\mu)-\frac{r_{j}-p_{j}}{2}} u_{k+1}^{P_{k+1}(\nu)} \cdots u_{n}^{P_{n}(\nu)} \bar{u}_{k+1}^{P_{k+1}(\mu)} \cdots \bar{u}_{n}^{P_{n}(\mu)} .
$$

If there are no $(\nu, \mu)$ with $c_{\nu, \mu} \neq 0$ and $\nu+\mu \notin \Delta$, then $\widetilde{R}(\boldsymbol{u}, \overline{\boldsymbol{u}}) \equiv 0$.

Note that it is possible that some of $P_{j}(\nu)-\frac{r_{j}+p_{j}}{2}, P_{j}(\mu)-\frac{r_{j}-p_{j}}{2}(j=1, \ldots, k)$ are negative integers. On the other hand, $P_{j}(\nu) \geq 0$ and $P_{j}(\mu) \geq 0$ for every $j(=k+1, \ldots, n)$. Set

$$
\begin{array}{llll}
a_{j}(\nu):=P_{j}(\nu)-\frac{r_{j}+p_{j}}{2}, & b_{j}(\mu):=P_{j}(\mu)-\frac{r_{j}-p_{j}}{2} & & \text { for each } j(=1, \ldots, k), \text { and } \\
a_{j}(\nu):=P_{j}(\nu), & b_{j}(\mu):=P_{j}(\mu) & \text { for each } j(=k+1, \ldots, n) .
\end{array}
$$

Then we have another expression:

$$
\widetilde{R}(\boldsymbol{u}, \overline{\boldsymbol{u}})=\sum_{\nu+\mu \notin \Delta} c_{\nu, \mu}\left(\prod_{j=1}^{k} u_{j}^{a_{j}(\nu)} \bar{u}_{j}^{b_{j}(\mu)}\right) \cdot u_{k+1}^{a_{k+1}(\nu)} \cdots u_{n}^{a_{n}(\nu)} \bar{u}_{k+1}^{b_{k+1}(\mu)} \cdots \bar{u}_{n}^{b_{n}(\mu)} .
$$

We now discuss the continuity of $\widetilde{R}(\boldsymbol{u}, \overline{\boldsymbol{u}})$. Let us pay attention to each "monomial"

$$
B_{\nu, \mu}(\boldsymbol{u}, \overline{\boldsymbol{u}}):=c_{\nu, \mu}\left(\prod_{j=1}^{k} u_{j}^{P_{j}(\nu)-\frac{r_{j}+p_{j}}{2}} \bar{u}_{j}^{P_{j}(\mu)-\frac{r_{j}-p_{j}}{2}}\right) \cdot u_{k+1}^{P_{k+1}(\nu)} \cdots u_{n}^{P_{n}(\nu)} \bar{u}_{k+1}^{P_{k+1}(\mu)} \cdots \bar{u}_{n}^{P_{n}(\mu)}, \quad c_{\nu, \mu} \neq 0
$$

of $\widetilde{R}(\boldsymbol{u}, \overline{\boldsymbol{u}})$. 
For each $j(=1, \ldots, k)$, we have

$$
a_{j}(\nu)+b_{j}(\mu)=P_{j}(\nu+\mu)-r_{j} .
$$

If $(\nu, \mu)$ satisfies $\nu+\mu \notin \Delta$, then there exists $j(=1, \ldots, k)$ such that $\nu+\mu \notin \Delta\left(P_{j}\right)$, and for such $j$, we have

$$
a_{j}(\nu)+b_{j}(\mu)=P_{j}(\nu+\mu)-r_{j} \geq 1 .
$$

Then, by Lemma 31, the function $u_{j}^{a_{j}(\nu)} \bar{u}_{j}^{b_{j}(\mu)}$ is of class $C^{\left(P_{j}(\nu+\mu)-r_{j}\right)-1}$ on $\mathbb{C}_{\sigma}^{n}$ if we define the value of the function $u_{j}^{a_{j}(\nu)} \bar{u}_{j}^{b_{j}(\mu)}$ at $u_{j}=0$ to be 0 . Especially, we see that $u_{j}^{a_{j}(\nu)} \bar{u}_{j}^{b_{j}(\mu)}$ is a continuous function on $\mathbb{C}_{\sigma}^{n}$.

On the other hand, if $(\nu, \mu)$ satisfies $\nu+\mu \in \Delta\left(P_{j}\right)$ for $j(=1, \ldots, k)$, then we have $a_{j}(\nu)+b_{j}(\mu)=$ $P_{j}(\nu+\mu)-r_{j}=0$, namely, $P_{j}(\nu+\mu)=r_{j}$, and also have $P_{j}(\nu-\mu)=p_{j}$ since the face function $f_{P_{j}}$ is a polar weighted homogeneous polynomial of polar degree $p_{j}$ with respect to $P_{j}$. Thus, for such $j$, we have

$$
a_{j}(\nu)=b_{j}(\mu)=0
$$

and hence, corresponding "monomial" $B_{\nu, \mu}(\boldsymbol{u}, \overline{\boldsymbol{u}})$ of $\widetilde{R}(\boldsymbol{u}, \overline{\boldsymbol{u}})$ does not contain such variables $u_{j}$ and $\bar{u}_{j}$.

Any way, for each "monomial" $B_{\nu, \mu}(\boldsymbol{u}, \overline{\boldsymbol{u}})$ of $\widetilde{R}(\boldsymbol{u}, \overline{\boldsymbol{u}})$, there exists $j(=1, \ldots, k)$ such that $\nu+\mu \notin \Delta\left(P_{j}\right)$ as stated above, and hence, we finally define

$$
B_{\nu, \mu}(\boldsymbol{u}, \overline{\boldsymbol{u}})=0
$$

when $u_{j}=0$ where $j=1, \ldots, k$ and $\nu+\mu \notin \Delta\left(P_{j}\right)$. For fixed $(\nu, \mu)$ with $c_{\nu, \mu} \neq 0$ and $\nu+\mu \notin \Delta$, we set

$$
\lambda_{\nu, \mu}(\tau):=\min \left\{P_{j}(\nu+\mu)-r_{j} \mid \nu+\mu \notin \Delta\left(P_{j}\right), j=1, \ldots, k\right\},
$$

where we know that $\left\{P_{j}(\nu+\mu)-r_{j} \mid \nu+\mu \notin \Delta\left(P_{j}\right), j=1, \ldots, k\right\} \neq \emptyset$. Since $P_{j}(\nu+\mu)-r_{j} \geq 1$ for every $j(=1, \ldots, k)$ with $\nu+\mu \notin \Delta\left(P_{j}\right)$, we see that $\lambda_{\nu, \mu}(\tau) \geq 1$. Thus, it is concluded that each "monomial" $B_{\nu, \mu}(\boldsymbol{u}, \overline{\boldsymbol{u}})$ of $\widetilde{R}(\boldsymbol{u}, \overline{\boldsymbol{u}})$ is of class $C^{\lambda_{\nu, \mu}(\tau)-1}$, and hence, continuous on $\mathbb{C}_{\sigma}^{n}$.

Recall that if there are no $(\nu, \mu)$ with $c_{\nu, \mu} \neq 0$ and $\nu+\mu \notin \Delta$, then $\widetilde{R}(\boldsymbol{u}, \overline{\boldsymbol{u}}) \equiv 0$. If there exists $(\nu, \mu)$ with $c_{\nu, \mu} \neq 0$ and $\nu+\mu \notin \Delta$, then we see $\left\{\lambda_{\nu, \mu}(\tau) \mid c_{\nu, \mu} \neq 0, \nu+\mu \notin \Delta\right\} \neq \emptyset$, and we conclude that

$$
\Lambda(\tau)=\min \left\{\lambda_{\nu, \mu}(\tau) \mid c_{\nu, \mu} \neq 0, \nu+\mu \notin \Delta\right\} .
$$

Note that $\Lambda(\tau) \geq 1$. Thus, $\widetilde{R}(\boldsymbol{u}, \overline{\boldsymbol{u}})=\sum_{\nu+\mu \notin \Delta} B_{\nu, \mu}(\boldsymbol{u}, \overline{\boldsymbol{u}})$ is of class $C^{\Lambda(\tau)-1}$, and hence, continuous on $\mathbb{C}_{\sigma}^{n}$. Note that

$$
\widetilde{R}(\underbrace{0, \ldots, 0}_{k}, \boldsymbol{u}^{\prime}, \overline{\boldsymbol{u}}^{\prime})=0 .
$$

We finally define the function $\tilde{f}(\boldsymbol{u}, \overline{\boldsymbol{u}})$ by the equality (8.6) again. Then, $\widetilde{f}(\boldsymbol{u}, \overline{\boldsymbol{u}})$ is a continuous function on $\mathbb{C}_{\sigma}^{n}$ and the strict transform $\tilde{V}$ of $V$ to $X$ in the toric chart $\mathbb{C}_{\sigma}^{n}$ is given as follows:

$$
\tilde{V}=\{\widetilde{f}(\boldsymbol{u}, \overline{\boldsymbol{u}})=0\} .
$$

We can also verify that

$$
\left\{\pi_{\sigma}^{*} f=0\right\}=\left\{u_{1}=0\right\} \cup \cdots \cup\left\{u_{k}=0\right\} \cup \tilde{V}
$$

in the toric chart $\mathbb{C}_{\sigma}^{n}$.

By (8.7), we have

$$
\begin{aligned}
\tilde{V}(\tau)^{*} \cap \mathbb{C}_{\sigma}^{n} & =\tilde{V} \cap\left\{u_{1}=\cdots=u_{k}=0, \text { and } u_{k+1} \neq 0, \cdots, u_{n} \neq 0\right\} \\
& =\left\{\left(\mathbf{0}, \boldsymbol{u}^{\prime}\right) \mid \widetilde{f}_{\Delta}\left(\boldsymbol{u}^{\prime}, \overline{\boldsymbol{u}}^{\prime}\right)=0, u_{k+1} \neq 0, \cdots, u_{n} \neq 0\right\} .
\end{aligned}
$$

Note that $\tilde{V}(\tau)^{*} \cap \mathbb{C}_{\sigma}^{n}$ is defined by $\widetilde{f}_{\Delta}\left(\boldsymbol{u}^{\prime}, \overline{\boldsymbol{u}}^{\prime}\right)$ only.

Recall that

$$
\pi_{\sigma}^{*} f_{\Delta}(\boldsymbol{u}, \overline{\boldsymbol{u}})=\prod_{j=1}^{k} u_{j}^{\frac{r_{j}+p_{j}}{2}} \bar{u}_{j}^{\frac{r_{j}-p_{j}}{2}} \times \widetilde{f}_{\Delta}\left(\boldsymbol{u}^{\prime}, \overline{\boldsymbol{u}}^{\prime}\right) .
$$


If $\left(\mathbf{0}, \boldsymbol{x}^{\prime}\right) \in \tilde{V}(\tau)^{*} \cap \mathbb{C}_{\sigma}^{n}$, then we have $\widetilde{f}_{\Delta}\left(\boldsymbol{x}^{\prime}, \overline{\boldsymbol{x}}^{\prime}\right)=0$,

$$
\hat{\boldsymbol{x}}:=(\underbrace{1, \ldots, 1}_{k}, \boldsymbol{x}^{\prime}) \in \mathbb{C}_{\sigma}^{* n},
$$

and $\pi_{\sigma}^{*} f_{\Delta}(\hat{\boldsymbol{x}})=\widetilde{f}_{\Delta}\left(\boldsymbol{x}^{\prime}, \overline{\boldsymbol{x}}^{\prime}\right)=0$ by (․8). By the Newton non-degeneracy assumption on the compact face $\Delta, 0$ is not a mixed critical value of the face function $f_{\Delta}: \mathbb{C}^{* n} \rightarrow \mathbb{C}$. Hence, $\hat{\boldsymbol{x}}$ is not a mixed critical point of $\pi_{\sigma}^{*} f_{\Delta}$.

For every $j(1 \leq j \leq k)$, we have

$$
\begin{aligned}
\frac{\partial \pi_{\sigma}^{*} f_{\Delta}}{\partial u_{j}} & =\frac{\partial}{\partial u_{j}}\left(\prod_{j=1}^{k} u_{j}^{\frac{r_{j}+p_{j}}{2}} \bar{u}_{j}^{\frac{r_{j}-p_{j}}{2}}\right) \times \widetilde{f}_{\Delta}\left(\boldsymbol{u}^{\prime}, \overline{\boldsymbol{u}}^{\prime}\right), \\
\frac{\partial \pi_{\sigma}^{*} f_{\Delta}}{\partial \bar{u}_{j}} & =\frac{\partial}{\partial \bar{u}_{j}}\left(\prod_{j=1}^{k} u_{j}^{\frac{r_{j}+p_{j}}{2}} \bar{u}_{j}^{\frac{r_{j}-p_{j}}{2}}\right) \times \widetilde{f}_{\Delta}\left(\boldsymbol{u}^{\prime}, \overline{\boldsymbol{u}}^{\prime}\right) .
\end{aligned}
$$

Hence, for $\hat{\boldsymbol{x}}=(\underbrace{1, \ldots, 1}_{k}, \boldsymbol{x}^{\prime})$, we have

$$
\begin{aligned}
& \frac{\partial \pi_{\sigma}^{*} f_{\Delta}}{\partial u_{j}}\left(1, \ldots, 1, \boldsymbol{x}^{\prime}\right)=(\cdots \cdots) \times \widetilde{f}_{\Delta}\left(\boldsymbol{x}^{\prime}, \overline{\boldsymbol{x}}^{\prime}\right)=0, \\
& \frac{\partial \pi_{\sigma}^{*} f_{\Delta}}{\partial \bar{u}_{j}}\left(1, \ldots, 1, \boldsymbol{x}^{\prime}\right)=(\cdots \cdots) \times \widetilde{f}_{\Delta}\left(\boldsymbol{x}^{\prime}, \overline{\boldsymbol{x}}^{\prime}\right)=0 .
\end{aligned}
$$

Since $\hat{\boldsymbol{x}}=(\underbrace{1, \ldots, 1}_{k}, \boldsymbol{x}^{\prime})$ is a mixed regular point of $\pi_{\sigma}^{*} f_{\Delta}$, we see that $\boldsymbol{x}^{\prime}$ is a mixed regular point of the mixed polynomial $\widetilde{f}_{\Delta}\left(\boldsymbol{u}^{\prime}, \overline{\boldsymbol{u}}^{\prime}\right)$. By this result and the implicit function theorem, $\tilde{V}(\tau)^{*} \cap \mathbb{C}_{\sigma}^{n}$ is a real analytic manifold of real dimension $2(n-k-1)$. This completes the proof of the assertion (i).

We next prove the assertion (ii). Take an arbitrary point

$$
\hat{\boldsymbol{u}}^{0} \in \tilde{V} \cap \hat{\pi}^{-1}(\mathbf{0})=\tilde{V} \cap \bigcup_{P \gg 0} \hat{E}(P) .
$$

By the Assumption $\left(^{*}\right)$, there exists an $n$-dimensional cone $\sigma=\left(P_{1}, \ldots, P_{n}\right)$ with strictly positive vertices $P_{1}, \ldots, P_{\kappa}(1 \leq \kappa \leq n)$ for which the toric chart $\mathbb{C}_{\sigma}^{n}$ contains $\hat{\boldsymbol{u}}^{0}$ and

$$
\hat{\boldsymbol{u}}^{0}=\boldsymbol{u}_{\sigma}^{0}=(\underbrace{0, \ldots, 0}_{\kappa}, u_{\kappa+1}^{0}, \ldots, u_{n}^{0}), u_{\kappa+1}^{0} \neq 0, \ldots, u_{n}^{0} \neq 0 \text { in } \mathbb{C}_{\sigma}^{n} .
$$

We now use the symbol $k$ instead of $\kappa$, and consider the $k$-dimensional cone

$$
\tau:=\left(P_{1}, \ldots, P_{k}\right) .
$$

Then we have $\boldsymbol{u}_{\sigma}^{0} \in \tilde{V}(\tau)^{*} \cap \mathbb{C}_{\sigma}^{n}$. We can use the arguments and some results in the proof of (i). Then, we see that $\left(u_{k+1}^{0}, \ldots, u_{n}^{0}\right)$ is a mixed regular point of the function $\widetilde{f}_{\Delta}\left(\boldsymbol{u}^{\prime}, \overline{\boldsymbol{u}}^{\prime}\right)$. Put

$$
N:=\sharp\left\{(\nu, \mu) \mid c_{\nu, \mu} \neq 0, \nu+\mu \notin \Delta\right\},
$$

and let the set

$$
\left\{\left(\nu_{\ell}, \mu_{\ell}\right) \mid \ell=1, \ldots, N\right\}
$$

coincide with the set $\{(\nu, \mu) \mid \nu+\mu \notin \Delta\}$. Then we obtain another expression:

$$
\widetilde{R}(\boldsymbol{u}, \overline{\boldsymbol{u}})=\sum_{\ell=1}^{N} c_{\ell}\left(\prod_{j=1}^{k} u_{j}^{a_{j}^{\ell}} \bar{u}_{j}^{b_{j}^{\ell}}\right)\left(\prod_{j=k+1}^{n} u_{j}^{a_{j}^{\ell}} \bar{u}_{j}^{b_{j}^{\ell}}\right) .
$$


Define a new polynomial with complex $N+(n-k)$ variables as follows:

$$
\widetilde{\widetilde{R}}\left(v_{1}, \ldots, v_{N}, \boldsymbol{u}^{\prime}\right):=\sum_{\ell=1}^{N} c_{\ell} v_{\ell}\left(\prod_{j=k+1}^{n} u_{j}^{a_{j}^{\ell}} \bar{u}_{j}^{b_{j}^{\ell}}\right) .
$$

We write $\boldsymbol{v}:=\left(v_{1}, \ldots, v_{N}\right)$, and define

$$
\widetilde{\widetilde{f}}\left(\boldsymbol{v}, \boldsymbol{u}^{\prime}\right):=\widetilde{f}_{\Delta}\left(\boldsymbol{u}^{\prime}, \overline{\boldsymbol{u}}^{\prime}\right)+\widetilde{\widetilde{R}}\left(\boldsymbol{v}, \boldsymbol{u}^{\prime}\right) .
$$

Now let $x_{j}$ and $y_{j}$, respectively, be the real part and the imaginary part of the variable $u_{j}$ for each $j(=k+1, \ldots, n)$. Since $\widetilde{\widetilde{R}}\left(\mathbf{0}, \boldsymbol{u}^{\prime}\right)=0$ where $\mathbf{0}=(\underbrace{0, \ldots, 0}_{N})$, the rank of the matrix

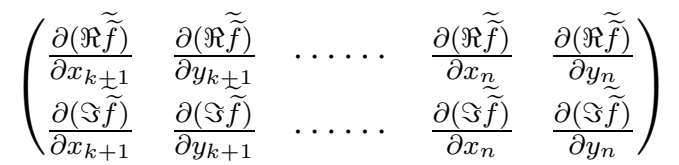

is 2 at $\left(\mathbf{0}, u_{k+1}^{0}, \ldots, u_{n}^{0}\right)$. Especially, we see that $\left(\mathbf{0}, u_{k+1}^{0}, \ldots, u_{n}^{0}\right)$ is a mixed regular point of the function $\widetilde{\widetilde{f}}\left(\boldsymbol{v}, \boldsymbol{u}^{\prime}\right)$. Hence, by the implicit function theorem, there exists a real analytic map

$$
F\left(\boldsymbol{v}, x_{k+1}, y_{k+1}, \ldots, \check{\xi}, \ldots, \check{\eta}, \ldots, x_{n}, y_{n}\right)
$$

to $\mathbb{R}^{2}$ such that

$$
\widetilde{\widetilde{f}}\left(\boldsymbol{v}, \boldsymbol{u}^{\prime}\right)=0 \Longleftrightarrow\left(\begin{array}{l}
\xi \\
\eta
\end{array}\right)=F\left(\boldsymbol{v}, x_{k+1}, y_{k+1}, \ldots, \check{\xi}, \ldots, \check{\eta}, \ldots, x_{n}, y_{n}\right)
$$

in some neighborhood of the point $\left(\mathbf{0}, u_{k+1}^{0}, \ldots, u_{n}^{0}\right)$, where $\mathbf{0}=(\underbrace{0, \ldots, 0}_{N})$. Here the notation $\check{\xi}, \ldots, \check{\eta}$ means we remove two real variables $\xi$ and $\eta$. Hence, we have

$$
\begin{gathered}
\widetilde{f}(\boldsymbol{u}, \overline{\boldsymbol{u}})=\widetilde{\widetilde{f}}\left(\left(\prod_{j=1}^{k} u_{j}^{a_{j}^{1}} \bar{u}_{j}^{b_{j}^{1}}\right), \ldots,\left(\prod_{j=1}^{k} u_{j}^{a_{j}^{N}} \bar{u}_{j}^{b_{j}^{N}}\right), \boldsymbol{u}^{\prime}\right)=0 \\
\Longleftrightarrow\left(\begin{array}{c}
\xi \\
\eta
\end{array}\right)=F\left(\left(\prod_{j=1}^{k} u_{j}^{a_{j}^{1}} \bar{u}_{j}^{b_{j}^{1}}\right), \ldots,\left(\prod_{j=1}^{k} u_{j}^{a_{j}^{N}} \bar{u}_{j}^{b_{j}^{N}}\right), x_{k+1}, y_{k+1}, \ldots, \check{\xi}, \ldots, \check{\eta}, \ldots, x_{n}, y_{n}\right)
\end{gathered}
$$

in some neighborhood of the point $\left(\mathbf{0}, u_{k+1}^{0}, \ldots, u_{n}^{0}\right)$, where $\mathbf{0}$ stands for $(\underbrace{0, \ldots, 0}_{k})$.

Suppose that $L\left(\Sigma^{*}\right)=\emptyset$. If $\tau$ is a cone of $\Sigma^{*}$ with only strictly positive vertices and $\tilde{V}(\tau)^{*} \cap \mathbb{C}_{\sigma}^{n} \neq \emptyset$ for some $n$-dimensional cone $\sigma$ of $\Sigma^{*}$ with $\tau \prec \sigma$, then we see that $\left\{P_{j}(\nu+\mu)-r_{j} \mid c_{\nu, \mu} \neq 0, \nu+\mu \notin\right.$ $\left.\Delta\left(P_{j}\right), j=1, \ldots, k\right\}=\emptyset$, where $k:=\operatorname{dim} \tau$. Hence, for every $(\nu, \mu)$ with $c_{\nu, \mu} \neq 0$, we have $\nu+\mu \in \Delta\left(P_{j}\right)$ for all $j=1, \ldots, k$, namely, $\nu+\mu \in \Delta$. Then we have $N=0$ and $\widetilde{R}(\boldsymbol{u}, \overline{\boldsymbol{u}}) \equiv 0$ by the argument in the proof of (i). Hence, $\tilde{V}(\tau)^{*} \cap \mathbb{C}_{\sigma}^{n}$ is a real analytic manifold. Thus, $\tilde{V}$ is a real analytic manifold.

Next suppose that $L\left(\Sigma^{*}\right) \neq \emptyset$. Then there exists a $k$-dimensional cone $\tau$ of $\Sigma^{*}$ with only strictly positive vertices, $\left\{P_{j}(\nu+\mu)-r_{j} \mid c_{\nu, \mu} \neq 0, \nu+\mu \notin \Delta\left(P_{j}\right), j=1, \ldots, k\right\} \neq \emptyset$, and $\tilde{V}(\tau)^{*} \cap \mathbb{C}_{\sigma}^{n} \neq \emptyset$ for some $n$-dimensional cone $\sigma$ of $\Sigma^{*}$ with $\tau \prec \sigma$. Then we have $N \geq 1$ and $\tilde{V}(\tau)^{*} \cap \mathbb{C}_{\sigma}^{n}$ is a $C^{\Lambda(\tau)-1}$-manifold by the argument in the proof of (i). Hence, $\tilde{V}$ is a $C^{\mathcal{L}-1}$-manifold. Moreover, it is easy to see that $\tilde{V}$ is a real analytic manifold outside of $\tilde{V} \cap \hat{\pi}^{-1}(\mathbf{0})$.

This completes the proof of the assertion (ii).

Corollary 33 (mixed homogeneous polynomial case, $n=2)$. Let us consider the case $n=2$. Let $(f, \mathbf{0})$ be the germ at $\mathbf{0} \in \mathbb{C}^{2}$ of a convenient and Newton non-degenerate strongly polar non-negative mixed weighted homogeneous polynomial $f(\boldsymbol{z}, \overline{\boldsymbol{z}})=\sum_{\nu, \mu} c_{\nu, \mu} \boldsymbol{z}^{\nu} \overline{\boldsymbol{z}}^{\mu}$ of radial degree $r$ with respect to the weight vector $P:={ }^{t}(1,1)$, and hence, $f(\boldsymbol{z}, \overline{\boldsymbol{z}})$ is a mixed homogeneous polynomial. We assume $f(\mathbf{0})=0$. Then, all

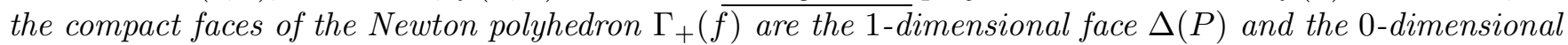
faces $\Delta\left(P+E_{1}\right), \Delta\left(P+E_{2}\right)$. By the assumption, we have $\operatorname{pdeg}_{P} f_{P}=\operatorname{pdeg}_{P} f \geq 0$. Then it is easy 
to check (Recall Lemma 29) that $\operatorname{rdeg}_{P+E_{i}} f_{P+E_{i}}=\operatorname{rdeg}_{P} f_{P+E_{i}}=\operatorname{rdeg}_{P} f_{P}=r$ and $\operatorname{pdeg}_{P+E_{i}} f_{P+E_{i}}=$ $\operatorname{pdeg}_{P} f_{P+E_{i}}=\operatorname{pdeg}_{P} f_{P} \geq 0$ for $i=1,2$. Hence, we can say that $(f, \mathbf{0})$ is of strongly polar non-negative mixed weighted homogeneous face type (Definition 12). In this case the (radial) dual Newton diagram $\Gamma^{*}(f)$ gives already a regular simplicial subdivision of $N_{\mathbb{R}}^{+}$which is admissible for $f$. Moreover, $\Gamma^{*}(f)$ is obviously convenient in the sense of Definition 24. Consider the toric modification $\hat{\pi}: X \rightarrow \mathbb{C}^{n}$ associated with $\Gamma^{*}(f)$. This is nothing but the "usual blowing-up". Let $(V, \mathbf{0})$ be the germ of the mixed hypersurface $V:=f^{-1}(0)$ at $\mathbf{0} \in \mathbb{C}^{2}$. We assume the Assumption $\left(^{*}\right)$. Since $\left\{P(\nu+\mu)-r \mid c_{\nu, \mu} \neq 0, \nu+\mu \notin \Delta(P)\right\}=\emptyset$, we have $L\left(\Sigma^{*}\right)=L\left(\Gamma^{*}(f)\right)=\emptyset$. By Theorem 32 (ii), we conclude that the strict transform germ $\tilde{V}$ at $\tilde{V} \cap \hat{\pi}^{-1}(\mathbf{0})$ is a real analytic manifold.

\section{The mixed polynomial $z_{1}^{4} \bar{z}_{1}^{2}+z_{1}^{a} \bar{z}_{1}^{4-a} z_{2}^{b} \bar{z}_{2}^{3-b}+\bar{z}_{1}^{2} z_{2}^{3}+z_{2}^{3} \bar{z}_{2}^{3}$}

As a typical example of Theorem [32, let us consider the following mixed polynomial of strongly polar non-negative mixed weighted homogeneous face type (see Definition [12)

$$
f(\boldsymbol{z}, \overline{\boldsymbol{z}}):=\left(z_{1}^{4}+z_{2}^{3}\right) \overline{\left(z_{1}^{2}+z_{2}^{3}\right)}
$$

Example 4.3 of [5], p.73 (see also [9]) describes a good resolution of the germ at $\mathbf{0}$ of the holomorphic polynomial

$$
\left(z_{1}^{4}+z_{2}^{3}\right)\left(z_{1}^{2}+z_{2}^{3}\right)
$$

Since $\left(z_{1}^{4}+z_{2}^{3}\right) \overline{\left(z_{1}^{2}+z_{2}^{3}\right)}$ and $\left(z_{1}^{4}+z_{2}^{3}\right)\left(z_{1}^{2}+z_{2}^{3}\right)$ have the same zero set and the same Newton polyhedron, we must be able to resolve the isolated singular point $\mathbf{0}$ of the germ $\left(\left(z_{1}^{4}+z_{2}^{3}\right) \overline{\left(z_{1}^{2}+z_{2}^{3}\right)}, \mathbf{0}\right)$ by the same toric modification. We have

$$
f(\boldsymbol{z}, \overline{\boldsymbol{z}})=\left(z_{1}^{4}+z_{2}^{3}\right) \overline{\left(z_{1}^{2}+z_{2}^{3}\right)}=z_{1}^{4} \bar{z}_{1}^{2}+z_{1}^{4} \bar{z}_{2}^{3}+\bar{z}_{1}^{2} z_{2}^{3}+z_{2}^{3} \bar{z}_{2}^{3} .
$$

The radial Newton polyhedron $\Gamma_{+}(f)$ of $(f, \mathbf{0})$ and its dual Newton diagram $\Gamma^{*}(f)$ are as in Figure 1, where we set

$$
E_{1}={ }^{t}(1,0), P={ }^{t}(3,2), Q={ }^{t}(3,4), E_{2}={ }^{t}(0,1) .
$$

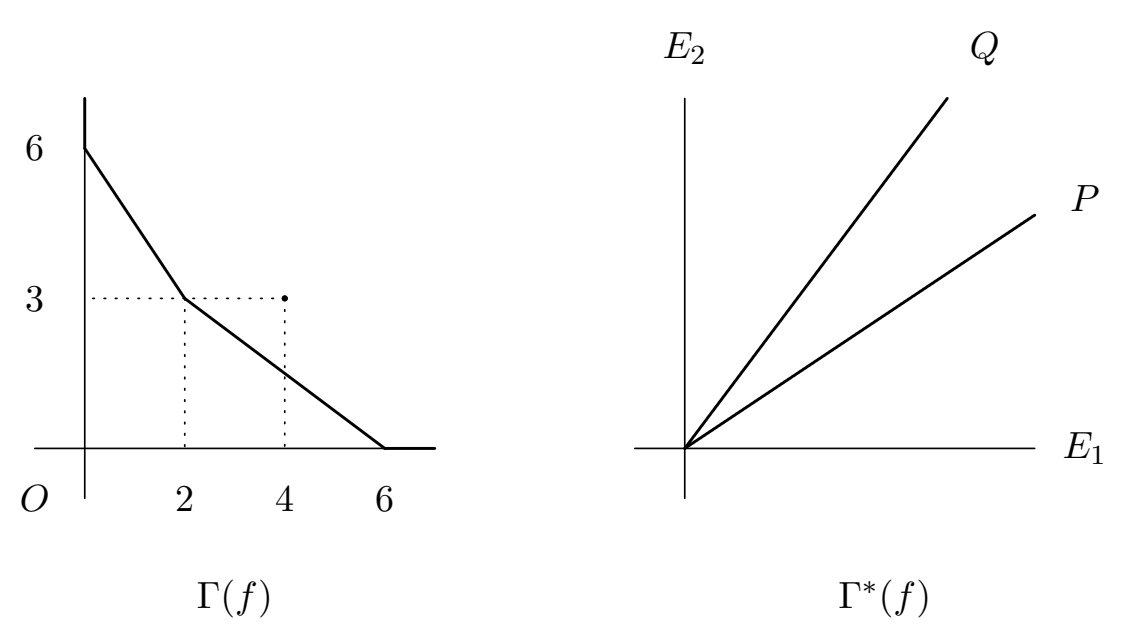

Figure 1. The radial Newton polyhedron $\Gamma_{+}(f)$ and the dual Newton diagram $\Gamma^{*}(f)$.

Figure 2 is a regular simplicial subdivision $\Sigma^{*}\left(\right.$ of $N_{\mathbb{R}}^{+}$) which is admissible for $f$, where we set

$$
R={ }^{t}(2,1), S={ }^{t}(1,1), T={ }^{t}(2,3), U={ }^{t}(1,2) .
$$

$\Sigma^{*}$ is called the canonical regular subdivision of $\Gamma^{*}(f)([1$, p.137) and it is convenient in the sense of Definition 24. 


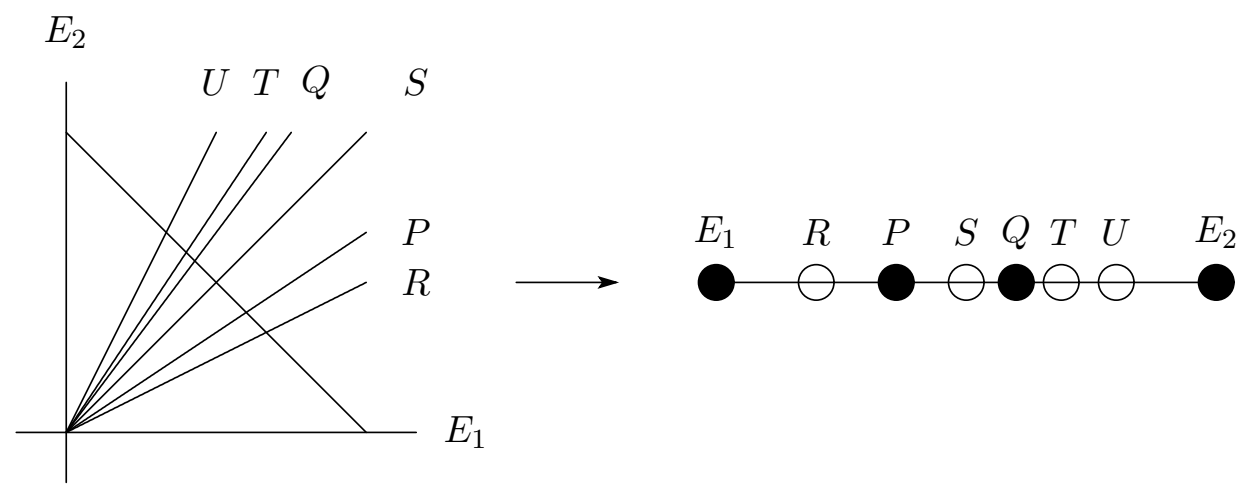

Figure 2. A regular simplicial subdivision $\Sigma^{*}$ which is admissible for $f$.

All the 2-dimensional cones (up to permutations of vertices) of $\Sigma^{*}$ are as follows:

$$
\begin{aligned}
& \tau_{1}:=\operatorname{Cone}\left(E_{1}, R\right)=\left(\begin{array}{ll}
1 & 2 \\
0 & 1
\end{array}\right), \quad \tau_{2}:=\operatorname{Cone}(R, P)=\left(\begin{array}{ll}
2 & 3 \\
1 & 2
\end{array}\right), \quad \tau_{3}:=\operatorname{Cone}(P, S)=\left(\begin{array}{ll}
3 & 1 \\
2 & 1
\end{array}\right), \\
& \tau_{4}:=\operatorname{Cone}(S, Q)=\left(\begin{array}{ll}
1 & 3 \\
1 & 4
\end{array}\right), \quad \tau_{5}:=\operatorname{Cone}(Q, T)=\left(\begin{array}{ll}
3 & 2 \\
4 & 3
\end{array}\right), \quad \tau_{6}:=\operatorname{Cone}(T, U)=\left(\begin{array}{ll}
2 & 1 \\
3 & 2
\end{array}\right), \\
& \text { and } \tau_{7}:=\operatorname{Cone}\left(U, E_{2}\right)=\left(\begin{array}{ll}
1 & 0 \\
2 & 1
\end{array}\right) .
\end{aligned}
$$

We consider the toric modification

$$
\hat{\pi}: X \rightarrow \mathbb{C}^{2}
$$

associated with $\Sigma^{*}$, which is called the canonical toric modification for $f([1$, p.138).

The face functions of the germ $(f, \mathbf{0})$ are

$$
f_{U}=f_{T}=z_{1}^{4} \bar{z}_{1}^{2}, \quad f_{S}=\bar{z}_{1}^{2} z_{2}^{3}, \quad f_{R}=z_{2}^{3} \bar{z}_{2}^{3}, \quad f_{Q}=z_{1}^{4} \bar{z}_{1}^{2}+\bar{z}_{1}^{2} z_{2}^{3}, \quad f_{P}=\bar{z}_{1}^{2} z_{2}^{3}+z_{2}^{3} \bar{z}_{2}^{3} .
$$

The radial and polar degrees of these face functions are calculated in Table 1 .

Let us discuss the Newton non-degeneracy of the germ $(f, \mathbf{0})$. For weight vectors $P={ }^{t}(3,2), Q={ }^{t}(3,4)$, $\operatorname{dim} \Delta(P)=\operatorname{dim} \Delta(Q)=1$, and

$$
f_{P}(\boldsymbol{z}, \overline{\boldsymbol{z}})=z_{2}^{3}{\overline{z_{1}}}^{2}+z_{2}^{3}{\overline{z_{2}}}^{3}, \quad f_{Q}(\boldsymbol{z}, \overline{\boldsymbol{z}})=z_{1}^{4}{\overline{z_{1}}}^{2}+z_{2}^{3}{\overline{z_{1}}}^{2} .
$$

For $P$, we have

Hence, we have

$$
\frac{\partial f_{P}}{\partial z_{1}}=0, \quad \frac{\partial f_{P}}{\partial \overline{z_{1}}}=2 z_{2}^{3} \overline{z_{1}}
$$

$$
\left|\frac{\partial f_{P}}{\partial z_{1}}\right| \neq\left|\frac{\partial f_{P}}{\partial \bar{z}_{1}}\right|
$$

on $\mathbb{C}^{* 2}$. Then by Corollary 3, $f_{P}$ has no mixed critical point on $\mathbb{C}^{* 2}$.

The function $f_{P}\left(z_{1}, 1\right)=\bar{z}_{1}^{2}+1$ is a surjective map from $\mathbb{C}^{*}$ onto $\mathbb{C} \backslash\{1\}$. Since $f_{P}\left(\sqrt{\frac{3}{2}}, \sqrt[3]{\frac{1}{2}}\right)=1$, we see $f_{P}: \mathbb{C}^{* 2} \rightarrow \mathbb{C}$ is surjective. Thus $(f, \mathbf{0})$ is strongly Newton non-degenerate over $\Delta(P)$.

For $Q$, we have

Hence, we also have

$$
\frac{\partial f_{Q}}{\partial z_{2}}=3 z_{2}^{2} \bar{z}_{1}^{2}, \quad \frac{\partial f_{Q}}{\partial \overline{z_{2}}}=0
$$

$$
\left|\frac{\partial f_{Q}}{\partial z_{2}}\right| \neq\left|\frac{\partial f_{Q}}{\partial \overline{z_{2}}}\right|
$$

on $\mathbb{C}^{* 2}$. Then by Corollary [3, $f_{Q}$ has no mixed critical point on $\mathbb{C}^{* 2}$.

The function $f_{Q}\left(1, z_{2}\right)=1+z_{2}^{3}$ is a surjective map from $\mathbb{C}^{*}$ onto $\mathbb{C} \backslash\{1\}$. Since $f_{Q}\left(\sqrt{\frac{1}{2}}, \sqrt[3]{\frac{7}{4}}\right)=1$, we see $f_{Q}: \mathbb{C}^{* 2} \rightarrow \mathbb{C}$ is surjective. Thus $(f, \mathbf{0})$ is strongly Newton non-degenerate over $\Delta(Q)$. 


\begin{tabular}{|c|c|c|}
\hline 0-dim face $\Delta(U)$ & \multicolumn{2}{|c|}{$f_{U}=z_{1}^{4}{\overline{z_{1}}}^{2}$} \\
\hline \multirow{2}{*}{ weight vector: } & 1 & $4+2,4-2$ \\
\cline { 2 - 3 } & 2 & $0+0,0-0$ \\
\hline radial degree & & 6 \\
\hline polar degree & & 2 \\
\hline
\end{tabular}

\begin{tabular}{|c|c|c|}
\hline 0-dim face $\Delta(T)$ & \multicolumn{2}{|c|}{$f_{T}=z_{1}^{4}{\overline{z_{1}}}^{2}$} \\
\hline weight vector: & 2 & $4+2,4-2$ \\
\cline { 2 - 3 } & 3 & $0+0,0-0$ \\
\hline radial degree & & 12 \\
\hline polar degree & & 4 \\
\hline
\end{tabular}

\begin{tabular}{|c|c|c|}
\hline 0-dim face $\Delta(S)$ & \multicolumn{2}{|c|}{$f_{S}=z_{2}^{3}{\overline{z_{1}}}^{2}$} \\
\hline \multirow{2}{*}{ weight vector: } & 1 & $0+2,0-2$ \\
\cline { 3 - 3 } & 1 & $3+0,3-0$ \\
\hline radial degree & & 5 \\
\hline polar degree & & 1 \\
\hline
\end{tabular}

\begin{tabular}{|c|c|c|}
\hline 0-dim face $\Delta(R)$ & \multicolumn{2}{|c|}{$f_{R}=z_{2}^{3}{\overline{z_{2}}}^{3}$} \\
\hline weight vector: & 2 & $0+0,0-0$ \\
\cline { 2 - 3 } & 1 & $3+3,3-3$ \\
\hline radial degree & & 6 \\
\hline polar degree & & 0 \\
\hline
\end{tabular}

\begin{tabular}{|c|c|c|c|}
\hline 1-dim face $\Delta(Q)$ & \multicolumn{3}{|c|}{$f_{Q}=z_{1}^{4}{\overline{z_{1}}}^{2}+z_{2}^{3}{\overline{z_{1}}}^{2}$} \\
\hline \multirow{2}{*}{ weight vector: } & 3 & $4+2,4-2$ & $0+2,0-2$ \\
\cline { 2 - 4 } & 4 & $0+0,0-0$ & $3+0,3-0$ \\
\hline & & the first term & the second term \\
\hline radial degree & & 18 & 18 \\
\hline polar degree & & 6 & 6 \\
\hline
\end{tabular}

\begin{tabular}{|c|c|c|c|}
\hline 1-dim face $\Delta(P)$ & \multicolumn{3}{|c|}{$f_{P}=z_{2}^{3}{\overline{z_{1}}}^{2}+z_{2}^{3}{\overline{z_{2}}}^{3}$} \\
\hline \multirow{2}{*}{ weight vector: } & 3 & $0+2,0-2$ & $0+0,0-0$ \\
\cline { 3 - 4 } & 2 & $3+0,3-0$ & $3+3,3-3$ \\
\hline & & the first term & the second term \\
\hline radial degree & & 12 & 12 \\
\hline polar degree & & 0 & 0 \\
\hline
\end{tabular}

TABLE 1. The face functions of the germ $\left(\left(z_{1}^{4}+z_{2}^{3} \overline{\left(z_{1}^{2}+z_{2}^{3}\right)}, \mathbf{0}\right)\right.$.

For 0-dimensional faces $\Delta(U)=\Delta(T)=\{(6,0)\}, \Delta(S)=\{(2,3)\}$ and $\Delta(R)=\{(0,6)\}$, the corresponding face functions are

$$
f_{U}=f_{T}=z_{1}^{4}{\overline{z_{1}}}^{2}, \quad f_{S}=z_{2}^{3}{\overline{z_{1}}}^{2}, \quad f_{R}=z_{2}^{3}{\overline{z_{2}}}^{3} .
$$

Since

$$
\begin{array}{ll}
\frac{\partial}{\partial z_{1}} z_{1}^{4}{\overline{z_{1}}}^{2}=4 z_{1}^{3}{\overline{z_{1}}}^{2}, & \frac{\partial}{\partial \overline{z_{1}}} z_{1}^{4}{\overline{z_{1}}}^{2}=2 z_{1}^{4} \overline{z_{1}}, \\
\frac{\partial}{\partial z_{1}} z_{2}^{3}{\overline{z_{1}}}^{2}=0, \quad \text { and } & \frac{\partial}{\partial \bar{z}_{1}} z_{2}^{3}{\overline{z_{1}}}^{2}=2 z_{2}^{3} \overline{z_{1}},
\end{array}
$$

$(f, \mathbf{0})$ is strongly Newton non-degenerate over $\Delta(U)=\Delta(T)$ and $\Delta(S)$.

However, the face function $z_{2}^{3}{\overline{z_{2}}}^{3}=\left|z_{2}\right|^{6}$ takes only real values. Hence, every point $\left(z_{1}, z_{2}\right) \in \mathbb{C}^{2}$ is a mixed critical point of $z_{2}^{3}{\overline{z_{2}}}^{3}$. Since $z_{2}^{3}{\overline{z_{2}}}^{3}=\left|z_{2}\right|^{6}>0$ on $\mathbb{C}^{* 2}, 0$ is not a mixed critical value of $z_{2}^{3}{\overline{z_{2}}}^{3}: \mathbb{C}^{* 2} \rightarrow \mathbb{C}$. Thus $(f, \mathbf{0})$ is not strongly Newton non-degenerate but Newton non-degenerate over $\Delta(R)$.

The second term $z_{1}^{4} \bar{z}_{2}^{3}$ of the mixed polynomial (9.1) belongs to the interior of the (radial) Newton polyhedron of $f(\boldsymbol{z}, \overline{\boldsymbol{z}})$. Let us replace $z_{1}^{4} \bar{z}_{2}^{3}$ by $z_{1}^{a} \bar{z}_{1}^{4-a} z_{2}^{b} \bar{z}_{2}^{3-b}$, and consider the mixed polynomials

$$
f_{a, b}(\boldsymbol{z}, \overline{\boldsymbol{z}}):=z_{1}^{4} \bar{z}_{1}^{2}+z_{1}^{a} \bar{z}_{1}^{4-a} z_{2}^{b} \bar{z}_{2}^{3-b}+\bar{z}_{1}^{2} z_{2}^{3}+z_{2}^{3} \bar{z}_{2}^{3},
$$

where $0 \leq a \leq 4,0 \leq b \leq 3$ are integers. Then, $\left(f_{a, b}, \mathbf{0}\right)$ is convenient.

Every mixed polynomial germ $\left(f_{a, b}, \mathbf{0}\right)$ has the same (radial) Newton polyhedron as $(f, \mathbf{0})$. Moreover, it has the same face functions as $(f, \mathbf{0})$. Hence, the germ $\left(f_{a, b}, \mathbf{0}\right)$ is also a Newton non-degenerate mixed polynomial germ of strongly polar non-negative mixed weighted homogeneous face type.

We put $f:=f_{a, b}$ hereafter.

Take a toric chart $\sigma=\operatorname{Cone}\left(P_{1}, P_{2}\right)$, where $P_{j}={ }^{t}\left(p_{1 j}, p_{2 j}\right)(j=1,2)$ and $P_{1}$ is strictly positive (namely, $P_{1} \neq E_{1}, E_{2}$. 
Then, for $\left(u_{1}, u_{2}\right) \in \mathbb{C}_{\sigma}^{2}$,

$$
\pi_{\sigma}\left(u_{1}, u_{2}\right)=\left(u_{1}^{p_{11}} u_{2}^{p_{12}}, u_{1}^{p_{21}} u_{2}^{p_{22}}\right)
$$

and we have

$$
\begin{aligned}
& \pi_{\sigma}^{*} f\left(u_{1}, u_{2}\right) \\
= & u_{1}^{4 p_{11}} \bar{u}_{1}^{2 p_{11}} u_{2}^{4 p_{12}}{\overline{u_{2}}}^{2 p_{12}}+u_{1}^{a p_{11}+b p_{21}} \bar{u}_{1}^{(4-a) p_{11}+(3-b) p_{21}} u_{2}^{a p_{12}+b p_{22}} \bar{u}_{2}(4-a) p_{12}+(3-b) p_{22} \\
& +u_{1}^{3 p_{21}} \bar{u}_{1}^{2 p_{11}} u_{2}^{3 p_{22}}{\overline{u_{2}}}^{2 p_{12}}+u_{1}^{3 p_{21}} \bar{u}_{1}^{3 p_{21}} u_{2}^{3 p_{22}}{\overline{u_{2}}}^{3 p_{22}} .
\end{aligned}
$$

We set $\tau:=$ Cone $\left(P_{1}\right)$.

We have

$$
f_{P_{1}}\left(z_{1}, z_{2}\right)=c_{1} z_{1}^{4}{\overline{z_{1}}}^{2}+c_{2}{\overline{z_{1}}}^{2} z_{2}^{3}+c_{3} z_{2}^{3}{\overline{z_{2}}}^{3}
$$

where

Hence, we have

$$
\left(c_{1}, c_{2}, c_{3}\right)= \begin{cases}(1,0,0) & \text { (if } \left.P_{1}=U \text { or } T\right) \\ (1,1,0) & \left(\text { if } P_{1}=Q\right) \\ (0,1,0) & \text { (if } \left.P_{1}=S\right) \\ (0,1,1) & \text { (if } \left.P_{1}=P\right) \\ (0,0,1) & \left(\text { if } P_{1}=R\right) .\end{cases}
$$

$$
\operatorname{rdeg} f_{P_{1}}=\left\{\begin{array}{ll}
6 p_{11} & \left(c_{1}=1\right) \\
2 p_{11}+3 p_{21} & \left(c_{2}=1\right) \\
6 p_{21} & \left(c_{3}=1\right)
\end{array} \quad \operatorname{pdeg} f_{P_{1}}= \begin{cases}2 p_{11} & \left(c_{1}=1\right) \\
-2 p_{11}+3 p_{21} & \left(c_{2}=1\right) \\
0 & \left(c_{3}=1\right)\end{cases}\right.
$$

and

$$
\begin{aligned}
& \frac{\operatorname{rdeg} f_{P_{1}}+\operatorname{pdeg} f_{P_{1}}}{2}= \begin{cases}4 p_{11} & \left(c_{1}=1\right) \\
3 p_{21} & \left(c_{2}=1 \text { or } c_{3}=1\right)\end{cases} \\
& \frac{\operatorname{rdeg} f_{P_{1}}-\operatorname{pdeg} f_{P_{1}}}{2}= \begin{cases}2 p_{11} & \left(c_{1}=1 \text { or } c_{2}=1\right) \\
3 p_{21} & \left(c_{3}=1\right) .\end{cases}
\end{aligned}
$$

The pull-back of the face function $f_{P_{1}}$ by $\pi_{\sigma}$ is as follows:

$$
\pi_{\sigma}^{*} f_{P_{1}}\left(u_{1}, u_{2}\right)=c_{1} u_{1}^{4 p_{11}}{\overline{u_{1}}}^{2 p_{11}} u_{2}^{4 p_{12}}{\overline{u_{2}}}^{2 p_{12}}+c_{2} u_{1}^{3 p_{21}} \bar{u}_{1}^{2 p_{11}} u_{2}^{3 p_{22}}{\overline{u_{2}}}^{2 p_{12}}+c_{3} u_{1}^{3 p_{21}}{\overline{u_{1}}}^{3 p_{21}} u_{2}^{3 p_{22}}{\overline{u_{2}}}^{3 p_{22}}
$$

Case $c_{1}=1$ : We have

$$
\begin{aligned}
\pi_{\sigma}^{*} f\left(u_{1}, u_{2}\right)= & u_{1}^{4 p_{11}} \bar{u}_{1}^{2 p_{11}}\left(u_{1}^{\left(3 p_{21}-4 p_{11}\right)} u_{2}^{3 p_{22} \bar{u}_{2}}{ }^{2 p_{12}}+u_{1}^{\left(3 p_{21}-4 p_{11}\right)} \bar{u}_{1}^{\left(3 p_{21}-2 p_{11}\right)} u_{2}^{3 p_{22}}{\overline{u_{2}}}^{3 p_{22}}\right. \\
& \left.+u_{2}^{4 p_{12}}{\overline{u_{2}}}^{2 p_{12}}+\underline{u}_{1}^{\left((a-4) p_{11}+b p_{21}\right)} \bar{u}_{1}^{\left((2-a) p_{11}+(3-b) p_{21}\right)} u_{2}^{\left(a p_{12}+b p_{22}\right)}{\overline{u_{2}}}^{\left((4-a) p_{12}+(3-b) p_{22}\right)}\right) .
\end{aligned}
$$

Here we have $6 p_{11}=2 p_{11}+3 p_{21}$ or $6 p_{11}<2 p_{11}+3 p_{21}$. Hence, $3 p_{21}-2 p_{11}>3 p_{21}-4 p_{11} \geq 0$.

Case $c_{2}=1$ : We have

$$
\begin{aligned}
\pi_{\sigma}^{*} f\left(u_{1}, u_{2}\right)= & u_{1}^{3 p_{21}} \bar{u}_{1}^{2 p_{11}}\left(u_{1}^{\left(4 p_{11}-3 p_{21}\right)} u_{2}^{4 p_{12}}{\overline{u_{2}}}^{2 p_{12}}+{\overline{u_{1}}}^{\left(3 p_{21}-2 p_{11}\right)} u_{2}^{3 p_{22}}{\overline{u_{2}}}^{3 p_{22}}\right. \\
& \left.+u_{2}^{3 p_{22}}{\overline{u_{2}}}^{2 p_{12}}+\underline{u_{1}^{\left(a p_{11}+(b-3) p_{21}\right)} \bar{u}_{1}^{\left((2-a) p_{11}+(3-b) p_{21}\right)} u_{2}^{\left(a p_{12}+b p_{22}\right)} \overline{u_{2}}\left((4-a) p_{12}+(3-b) p_{22}\right)}\right) .
\end{aligned}
$$

Here we have $2 p_{11}+3 p_{21} \leq 6 p_{11}$ and $2 p_{11}+3 p_{21} \leq 6 p_{21}$. Hence, $4 p_{11}-3 p_{21} \geq 0$ and $3 p_{21}-2 p_{11} \geq 0$.

Case $c_{3}=1$ : We have

$$
\begin{aligned}
\pi_{\sigma}^{*} f\left(u_{1}, u_{2}\right)= & u_{1}^{3 p_{21}} \bar{u}_{1}^{3 p_{21}}\left({\overline{u_{1}}}^{\left(2 p_{11}-3 p_{21}\right)} u_{2}^{3 p_{22}}{\overline{u_{2}}}^{2 p_{12}}+u_{1}^{\left(4 p_{11}-3 p_{21}\right)}{\overline{u_{1}}}^{\left(2 p_{11}-3 p_{21}\right)} u_{2}^{3 p_{22}}{\overline{u_{2}}}^{3 p_{22}}\right. \\
& \left.+u_{2}^{3 p_{22} \bar{u}_{2}}{ }^{3 p_{22}}+\underline{u}_{1}^{\left(a p_{11}+(b-3) p_{21}\right)} \bar{u}_{1}^{\left((4-a) p_{11}-b p_{21}\right)} u_{2}^{\left(a p_{12}+b p_{22}\right)} \bar{u}_{2}^{\left((4-a) p_{12}+(3-b) p_{22}\right)}\right) .
\end{aligned}
$$

Here we have $6 p_{21} \leq 2 p_{11}+3 p_{21}$. Hence, $2 p_{11}-3 p_{21} \geq 0$ and $4 p_{11}-3 p_{21} \geq 2 p_{11}>0$. 
For the exponents of the underlined terms above, we calculate

$$
\begin{aligned}
& c_{1}=1 \quad \Longrightarrow \quad\left((a-4) p_{11}+b p_{21}\right)+\left((2-a) p_{11}+(3-b) p_{21}\right)=-2 p_{11}+3 p_{21} \geq 2 p_{11} \geq 2 \\
& c_{2}=1 \quad \Longrightarrow \quad\left(a p_{11}+(b-3) p_{21}\right)+\left((2-a) p_{11}+(3-b) p_{21}\right)=2 p_{11} \geq 2 \\
& c_{3}=1 \quad \Longrightarrow \quad\left(a p_{11}+(b-3) p_{21}\right)+\left((4-a) p_{11}-b p_{21}\right)=4 p_{11}-3 p_{21} \geq 2 p_{11} \geq 2 .
\end{aligned}
$$

Hence, we may define the value of each underlined term at $\left(0, u_{2}\right)$ to be 0 . Then each underlined term is of class $C^{1}$. Hence $\tilde{R}, \tilde{f}$ are also of class $C^{1}$.

Let us consider the case $P_{1}=R={ }^{t}(2,1)$. Namely, $\left(c_{1}, c_{2}, c_{3}\right)=(0,0,1)$ and $\left(p_{11}, p_{21}\right)=(2,1)$. Recall that $f_{R}=z_{2}^{3} \bar{z}_{2}^{3}$. We have

$$
\operatorname{rdeg} f_{P_{1}}=6 \text { and } \operatorname{pdeg} f_{P_{1}}=0,
$$

and hence,

$$
\frac{\operatorname{rdeg} f_{P_{1}}+\operatorname{pdeg} f_{P_{1}}}{2}=3 \quad \text { and } \quad \frac{\operatorname{rdeg} f_{P_{1}}-\operatorname{pdeg} f_{P_{1}}}{2}=3
$$

We have

$$
\begin{aligned}
& \pi_{\sigma}^{*} f\left(u_{1}, u_{2}\right)=u_{1}^{3}{\overline{u_{1}}}^{3}\left({\overline{u_{1}}}^{(4-3)} u_{2}^{3 p_{22}}{\overline{u_{2}}}^{2 p_{12}}+u_{1}^{(8-3)}{\overline{u_{1}}}^{(4-3)} u_{2}^{3 p_{22}}{\overline{u_{2}}}^{3 p_{22}}\right. \\
& +u_{2}^{3 p_{22}} \bar{u}_{2}^{3 p_{22}}+\underline{\left.u_{1}^{(2 a+(b-3))} \bar{u}_{1}^{(2(4-a)-b)} u_{2}^{\left(a p_{12}+b p_{22}\right)} \bar{u}_{2}^{\left((4-a) p_{12}+(3-b) p_{22}\right)}\right)} \\
& =u_{1}^{3}{\overline{u_{1}}}^{3}\left(\overline{u_{1}} u_{2}^{3 p_{22}}{\overline{u_{2}}}^{2 p_{12}}+u_{1}^{5} \overline{u_{1}} u_{2}^{3 p_{22}}{\overline{u_{2}}}^{3 p_{22}}\right. \\
& +u_{2}^{3 p_{22}}{\overline{u_{2}}}^{3 p_{22}}+\underline{\left.u_{1}^{(2 a+(b-3))} \bar{u}_{1}^{(2(4-a)-b)} u_{2}^{\left(a p_{12}+b p_{22}\right)} \bar{u}_{2}^{\left((4-a) p_{12}+(3-b) p_{22}\right)}\right)}
\end{aligned}
$$

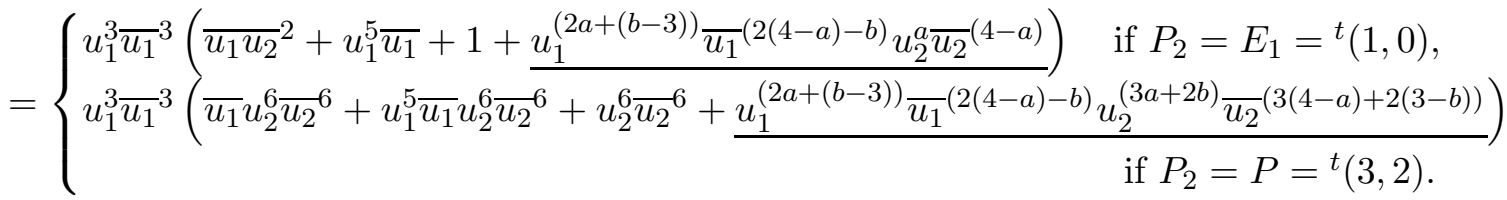

Hence, we have

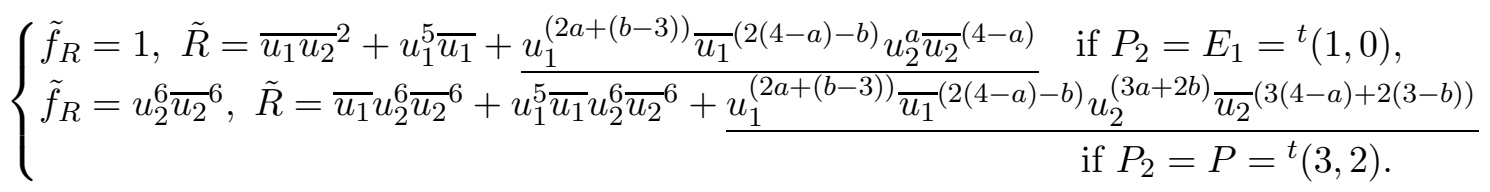

Thus we have $\tilde{f}\left(0, u_{2}\right) \neq 0$ if $u_{2} \neq 0$. Hence, we see that $\tilde{V}(R)^{*}=\emptyset$.

Moreover, in the toric chart $\sigma=\operatorname{Cone}\left(R, E_{1}\right)$, we also see that $\tilde{V}=\emptyset$ in some neighborhood of $\hat{E}(R)$ since $\tilde{f}\left(0, u_{2}\right)=\tilde{f}_{R}\left(0, u_{2}\right)=1$.

Next let us consider the case $P_{1}=U={ }^{t}(1,2)$. Namely, $\left(c_{1}, c_{2}, c_{3}\right)=(1,0,0)$ and $\left(p_{11}, p_{21}\right)=(1,2)$. Recall that $f_{U}=z_{1}^{4}{\overline{z_{1}}}^{2}$. We have

$$
\operatorname{rdeg} f_{P_{1}}=6 \text { and } \operatorname{pdeg} f_{P_{1}}=2,
$$

and hence,

We have

$$
\frac{\operatorname{rdeg} f_{P_{1}}+\operatorname{pdeg} f_{P_{1}}}{2}=4 \text { and } \frac{\operatorname{rdeg} f_{P_{1}}-\operatorname{pdeg} f_{P_{1}}}{2}=2 .
$$

$$
\begin{aligned}
& \pi_{\sigma}^{*} f\left(u_{1}, u_{2}\right)=u_{1}^{4}{\overline{u_{1}}}^{2}\left(u_{1}^{(6-4)} u_{2}^{3 p_{22}}{\overline{u_{2}}}^{2 p_{12}}+u_{1}^{(6-4)}{\overline{u_{1}}}^{(6-2)} u_{2}^{3 p_{22}}{\overline{u_{2}}}^{3 p_{22}}\right. \\
& +u_{2}^{4 p_{12}}{\overline{u_{2}}}^{2 p_{12}}+\underline{\left.u_{1}^{((a-4)+2 b)} \bar{u}_{1}^{((2-a)+(3-b) \times 2)} u_{2}^{\left(a p_{12}+b p_{22}\right)} \bar{u}_{2}^{\left((4-a) p_{12}+(3-b) p_{22}\right)}\right)}
\end{aligned}
$$

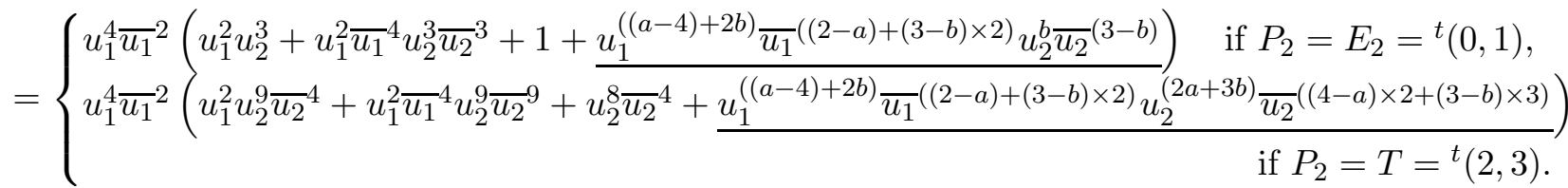


Hence, we have

$$
\left\{\begin{array}{l}
\tilde{f}_{U}=1, \tilde{R}=u_{1}^{2} u_{2}^{3}+u_{1}^{2}{\overline{u_{1}}}^{4} u_{2}^{3}{\overline{u_{2}}}^{3}+u_{1}^{((a-4)+2 b)} \bar{u}_{1}^{((2-a)+(3-b) \times 2)} u_{2}^{b}{\overline{u_{2}}}^{(3-b)} \quad \text { if } P_{2}=E_{2}={ }^{t}(0,1), \\
\tilde{f}_{U}=u_{2}^{8}{\overline{u_{2}}}^{4}, \tilde{R}=u_{1}^{2} u_{2}^{9}{\overline{u_{2}}}^{4}+u_{1}^{2}{\overline{u_{1}}}^{4}{\overline{u_{2}^{9}}}_{\bar{u}_{2}}{ }^{9}+{\underline{u_{1}^{((a-4)+2 b)}} \overline{u_{1}((2-a)+(3-b) \times 2)} u_{2}^{(2 a+3 b)} \overline{u_{2}}((4-a) \times 2+(3-b) \times 3)}^{\text {if } P_{2}=T={ }^{t}(2,3) .}
\end{array}\right.
$$

Thus we have $\tilde{f}\left(0, u_{2}\right) \neq 0$ if $u_{2} \neq 0$. Hence, we see that $\tilde{V}(U)^{*}=\emptyset$.

Moreover, in the toric chart $\sigma=\operatorname{Cone}\left(U, E_{2}\right)$, we also see that $\tilde{V}=\emptyset$ in some neighborhood of $\hat{E}(U)$ since $\tilde{f}\left(0, u_{2}\right)=\tilde{f}_{U}\left(0, u_{2}\right)=1$.

Now take a toric chart $\tau:=\operatorname{Cone}\left(P_{1}, P_{2}\right)$, where $P_{j}={ }^{t}\left(p_{1 j}, p_{2 j}\right)(j=1,2)$, and both $P_{1}$ and $P_{2}$ are strictly positive weight vectors (namely, $P_{j} \neq E_{1}, E_{2}(j=1,2)$ ).

We define the toric chart $\sigma:=\tau$.

Then there are five such 2-dimentional charts $\sigma$ as follows (recall the notation (9.2)):

$$
\begin{array}{ll}
\tau_{2}=\text { Cone }(R, P), & \pi_{\tau_{2}}\left(u_{1}, u_{2}\right)=\left(u_{1}^{2} u_{2}^{3}, u_{1} u_{2}^{2}\right), \\
\tau_{3}=\text { Cone }(P, S), & \pi_{\tau_{3}}\left(u_{1}, u_{2}\right)=\left(u_{1}^{3} u_{2}, u_{1}^{2} u_{2}\right), \\
\tau_{4}=\text { Cone }(S, Q), & \pi_{\tau_{4}}\left(u_{1}, u_{2}\right)=\left(u_{1} u_{2}^{3}, u_{1} u_{2}^{4}\right), \\
\tau_{5}=\text { Cone }(Q, T), & \pi_{\tau_{5}}\left(u_{1}, u_{2}\right)=\left(u_{1}^{3} u_{2}^{2}, u_{1}^{4} u_{2}^{3}\right), \\
\tau_{6}=\text { Cone }(T, U), & \pi_{\tau_{6}}\left(u_{1}, u_{2}\right)=\left(u_{1}^{2} u_{2}, u_{1}^{3} u_{2}^{2}\right) .
\end{array}
$$

Hence, we have

$$
\begin{aligned}
& \pi_{\tau_{2}}^{*} f\left(u_{1}, u_{2}\right)=u_{1}^{8} u_{2}^{12}{\overline{u_{1}}}^{4}{\overline{u_{2}}}^{6}+u_{1}^{2 a+b} u_{2}^{3 a+2 b}{\overline{u_{1}}}^{11-2 a-b}{\overline{u_{2}}}^{18-3 a-2 b}+u_{1}^{3} u_{2}^{6}{\overline{u_{1}}}^{4}{\overline{u_{2}}}^{6}+u_{1}^{3} u_{2}^{6}{\overline{u_{1}}}^{3}{\overline{u_{2}}}^{6}, \\
& \pi_{\tau_{3}}^{*} f\left(u_{1}, u_{2}\right)=u_{1}^{12} u_{2}^{4}{\overline{u_{1}}}^{6}{\overline{u_{2}}}^{2}+u_{1}^{3 a+2 b} u_{2}^{a+b} \bar{u}_{1}^{18-3 a-2 b} \bar{u}^{7}{ }^{7-a-b}+u_{1}^{6} u_{2}^{3}{\overline{u_{1}}}^{6}{\overline{u_{2}}}^{2}+u_{1}^{6} u_{2}^{3}{\overline{u_{1}}}^{6}{\overline{u_{2}}}^{3}, \\
& \pi_{\tau_{4}}^{*} f\left(u_{1}, u_{2}\right)=u_{1}^{4} u_{2}^{12}{\overline{u_{1}}}^{2}{\overline{u_{2}}}^{6}+u_{1}^{a+b} u_{2}^{3 a+4 b} \bar{u}_{1}^{7-a-b} \bar{u}_{2}^{24-3 a-4 b}+u_{1}^{3} u_{2}^{12}{\overline{u_{1}}}^{2}{\overline{u_{2}}}^{6}+u_{1}^{3} u_{2}^{12}{\overline{u_{1}}}^{3}{\overline{u_{2}}}^{12}, \\
& \pi_{\tau_{5}}^{*} f\left(u_{1}, u_{2}\right)=u_{1}^{12} u_{2}^{8}{\overline{u_{1}}}^{6}{\overline{u_{2}}}^{4}+u_{1}^{3 a+2 b} u_{2}^{2 a+3 b}{\overline{u_{1}}}^{24-3 a-4 b}{\overline{u_{2}}}^{17-2 a-3 b}+u_{1}^{12} u_{2}^{9}{\overline{u_{1}}}^{6}{\overline{u_{2}}}^{4}+u_{1}^{12} \bar{u}_{2}^{9}{\overline{u_{1}}}^{12}{\overline{u_{2}}}^{9}, \\
& \pi_{\tau_{6}}^{*} f\left(u_{1}, u_{2}\right)=u_{1}^{8} u_{2}^{4}{\overline{u_{1}}}^{4}{\overline{u_{2}}}^{2}+u_{1}^{2 a+3 b} u_{2}^{a+2 b} \bar{u}_{1}^{17-2 a-3 b} \bar{u}^{10-a-2 b}+u_{1}^{9} \bar{u}_{2}^{6}{\overline{u_{1}}}^{4}{\overline{u_{2}}}^{2}+u_{1}^{9} \bar{u}_{2}^{6}{\overline{u_{1}}}^{9}{\overline{u_{2}}}^{6},
\end{aligned}
$$

and

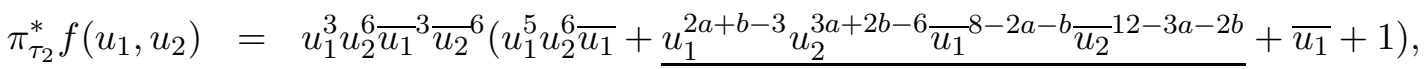

$$
\begin{aligned}
& \pi_{\tau_{3}}^{*} f\left(u_{1}, u_{2}\right)=u_{1}^{6} u_{2}^{3}{\overline{u_{1}}}^{6}{\overline{u_{2}}}^{2}\left(u_{1}^{6} u_{2}+u_{1}^{3 a+2 b-6} u_{2}^{a+b-3}{\overline{u_{1}}}^{12-3 a-2 b}{\overline{u_{2}}}^{5-a-b}+1+\overline{u_{2}}\right), \\
& \pi_{\tau_{4}}^{*} f\left(u_{1}, u_{2}\right)=u_{1}^{3} u_{2}^{12}{\overline{u_{1}}}^{2}{\overline{u_{2}}}^{6}\left(u_{1}+u_{1}^{a+b-3} u_{2}^{3 a+4 b-12}{\overline{u_{1}}}^{5-a-b}{\overline{u_{2}}}^{18-3 a-4 b}+1+{\overline{u_{1} u_{2}}}^{6}\right),
\end{aligned}
$$

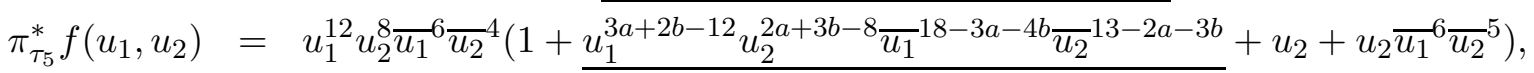

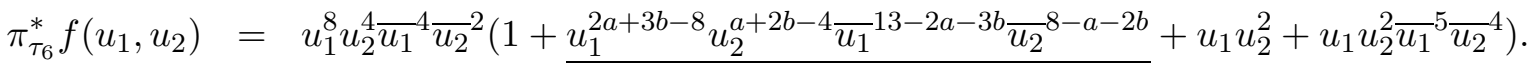

For the exponents of the underlined terms, we calculate

$$
\begin{array}{lll}
(2 a+b-3)+(8-2 a-b)=5, & & (3 a+2 b-6)+(12-3 a-2 b)=6, \\
(3 a+2 b-6)+(12-3 a-2 b)=6, & (a+b-3)+(5-a-b)=2, \\
(a+b-3)+(5-a-b)=2, & (3 a+4 b-12)+(18-3 a-4 b)=6, \\
(3 a+2 b-12)+(18-3 a-4 b)=6, & (2 a+3 b-8)+(13-2 a-3 b)=5, \\
(2 a+3 b-8)+(13-2 a-3 b)=5, & (a+2 b-4)+(8-a-2 b)=4 .
\end{array}
$$

Hence, we define the value of each underlined term as follows: If the exponent of $u_{1}$ or $\overline{u_{1}}$ is negative, then we define the value of the term at $\left(0, u_{2}\right)$ to be 0 . If the exponent of $u_{2}$ or $\overline{u_{2}}$ is negative, then we define the value of the term at $\left(u_{1}, 0\right)$ to be 0 .

Then we see that all underlined terms are of class $C^{1}$. Hence, $\tilde{R}$ and $\tilde{f}$ are also of class $C^{1}$.

However, by the above calculations, we have $\tilde{f}(0,0) \neq 0$, and we conclude that $\tilde{V}(\tau)^{*}=\emptyset$ in some neighborhood of $\left(u_{1}, u_{2}\right)=(0,0)$.

By the above results, we see that $f$ and $\Sigma^{*}$ satisfy Assumption $\left(^{*}\right)$ in the statement of Theorem 32 , Hence, by Theorem 32, The strict transform $\tilde{V}$ is a $C^{1}$-manifold in some neighborhood of $\hat{\pi}^{-1}(\mathbf{0}) \cap \tilde{V}$ for every $(a, b)$ with $0 \leq a \leq 4$ and $0 \leq b \leq 3$ and a real analytic manifold outside of $\hat{\pi}^{-1}(\mathbf{0}) \cap \tilde{V}$. 
We now summarize the contents of this section as follows:

Each of mixed polynomial germs

$$
f_{a, b}(\boldsymbol{z}, \overline{\boldsymbol{z}}):=z_{1}^{4} \bar{z}_{1}^{2}+z_{1}^{a} \bar{z}_{1}^{4-a} z_{2}^{b} \bar{z}_{2}^{3-b}+\bar{z}_{1}^{2} z_{2}^{3}+z_{2}^{3} \bar{z}_{2}^{3}
$$

at $\mathbf{0} \in \mathbb{C}^{2}$, where $0 \leq a \leq 4,0 \leq b \leq 3$ are integers, has five face functions. The four face functions

$$
\left(f_{a, b}\right)_{U}=\left(f_{a, b}\right)_{T}=z_{1}^{4} \bar{z}_{1}^{2}, \quad\left(f_{a, b}\right)_{S}=\bar{z}_{1}^{2} z_{2}^{3}, \quad\left(f_{a, b}\right)_{Q}=z_{1}^{4} \bar{z}_{1}^{2}+\bar{z}_{1}^{2} z_{2}^{3}, \quad\left(f_{a, b}\right)_{P}=\bar{z}_{1}^{2} z_{2}^{3}+z_{2}^{3} \bar{z}_{2}^{3}
$$

are strongly polar non-negative mixed weighted homogeneous polynomials. Especially, the two face functions $\left(f_{a, b}\right)_{P}=\bar{z}_{1}^{2} z_{2}^{3}+z_{2}^{3} \bar{z}_{2}^{3}$ and $\left(f_{a, b}\right)_{R}=z_{2}^{3} \bar{z}_{2}^{3}$ are strongly mixed weighted homogeneous polynomials of polar degree 0. Moreover, $\left(f_{a, b}, \mathbf{0}\right)$ is strongly Newton non-degenerate over $\Delta(U)=\Delta(T), \Delta(S), \Delta(Q)$ and $\Delta(P)$, however, it is not strongly Newton non-degenerate but Newton non-degenerate over $\Delta(R)$.

Let $V:=f_{a, b}^{-1}(0)$ be the germ of the mixed hypersurface at $\mathbf{0} \in \mathbb{C}^{2}$ and $\tilde{V}$ be the strict transform of $V$ to $X$ via the canonical toric modification (9.3). Then, $\left(f_{a, b}, \mathbf{0}\right), \Sigma^{*}$ and $\tilde{V}$ satisfy the assumptions of Theorem 32. By Theorem 32, the strict transform $\tilde{V}$ is a $C^{1}$-manifold in some neighborhood of $\hat{\pi}^{-1}(\mathbf{0}) \cap \tilde{V}$ and a real analytic manifold outside of $\hat{\pi}^{-1}(\mathbf{0}) \cap \tilde{V}$ for every $(a, b)$ with $0 \leq a \leq 4$ and $0 \leq b \leq 3$.

\section{Acknowledgments.}

The authors would like to thank the referee for careful readings and many good advices, Professor Mutsuo Oka (Tokyo University of Science) for his kind and good advices, and Professor Toru Ohmoto (Hokkaido University) for introducing them studies of mixed functions.

\section{REFERENCES}

[1] M. OKA, Non-degenerate complete intersection singularity, Hermann, Paris, 1997.

[2] M. OKA, Topology of polar weighted homogeneous hypersurfaces, Kodai Math. J. 31 (2008), $163-182$.

[3] M. OkA, Non-degenerate mixed functions, Kodai Math. J. 33 (2010), 1-62.

[4] M. OKA, Mixed functions of strongly polar weighted homogeneous face type, Singularities in Geometry and Topology 2011, Advanced Study in Pure Math. 66 (2015), 173-202.

[5] M. OKA, Introduction to Complex and Mixed hypersurface singularities (in Japanese), Maruzen, Tokyo, 2018.

[6] A. Pichon, Real analytic germs $f \bar{g}$ and open-book decompositions of the 3-sphere, Internat. J. Math., 16 (2005), 1-12.

[7] A. Pichon and J. Seade, Fibered multilinks and singularities $f \bar{g}$, Math. Ann. 342 (2008), 487-514.

[8] A. Pichon and J. Seade, Milnor fibrations and the Thom property for maps $f \bar{g}$, J. of Singularities 3 (2011), 144-150.

[9] S. Saito And K. TAKashimizu, Good resolutions of germs of plane curves and complex surfaces (in Japanese), Hokkaido Kyoikudaigaku Kiyo $\mathbf{7 1}$ (2021), 1-16.

[10] S. Saito and K. Takashimizu, Newton Non-degeneracy of Mixed Functions (in Japanese), preprint, 2021.

[11] K. TAKashimizu, Toric resolutions of mixed hypersurface singularities (in Japanese), Master's thesis, Hokkaido University of Education, 2021.

[12] A. N. VArchenko, Zeta-function of monodromy and Newton's diagram, Invent. Math. 37 (1976), 253-262.

Department of Mathematics Education, Asahikawa Campus, Hokkaido University of Education, Asahikawa 070-8621, JAPAN

Email address: saito.sachiko@a.hokkyodai.ac.jp 